Article

\title{
Ocean Surface Cross Section for Bistatic HF Radar Incorporating a Six DOF Oscillation Motion Model
}

\author{
Guowei Yao ${ }^{1}$, Junhao Xie ${ }^{1, *}$ and Weimin Huang ${ }^{2}$ (I) \\ 1 Department of Electronic Engineering, Harbin Institute of Technology, Harbin 150001, China; \\ yaoguoweiygw@hit.edu.cn \\ 2 Faculty of Engineering and Applied Science, Memorial University of Newfoundland, St. Johns, NL A1B 3X5, \\ Canada; weimin@mun.ca \\ * Correspondence: xj@hit.edu.cn
}

Received: 29 September 2019; Accepted: 19 November 2019; Published: 21 November 2019

\begin{abstract}
To investigate the characteristics of sea clutter, based on ocean surface electromagnetic scattering theory, the first- and second-order ocean surface scattering cross sections for bistatic high-frequency (HF) radar incorporating a multi-frequency six degree-of-freedom (DOF) oscillation motion model are mathematically derived. The derived radar cross sections (RCSs) can be reduced to the floating platform based monostatic case or onshore bistatic case for corresponding geometry setting. Simulation results show that the six DOF oscillation motion will result in more additional peaks in the radar Doppler spectra and the amplitudes and frequencies of these motion-induced peaks are decided by the amplitudes and frequencies of the oscillation motion. The effect of the platform motion on the first-order radar spectrum is greater than that of the second-order, and the motion-induced peaks in the first-order spectrum may overlap with the second-order spectrum. Furthermore, yaw is the dominant factor affecting the radar spectra, especially the second-order. Moreover, the effect of platform motion on radar spectra and the amplitudes of the second-order spectrum decreases as the bistatic angle increases. In addition, it should be noted that the amplitudes of the Bragg peaks may be lower than those of the motion-induced peaks due to the low frequency (LF) oscillation motion of the floating platform, which is an important finding for the applications of the floating platform based bistatic HF radar in moving target detection and ocean surface dynamics parameter estimation.
\end{abstract}

Keywords: bistatic HF radar; radar cross section (RCS); sea clutter

\section{Introduction}

High-frequency (HF) radar has been successfully deployed to detect ocean surface moving target and remote sensing of ocean surface dynamics such as wind direction and speed, current and wave parameters in many countries for decades [1-13] because it can provide real-time, all-weather surveillance beyond the horizon. Based on the geometry, HF radar can be generally divided into monostatic (transmitter and receiver are collocated) and bistatic (transmitter and receiver are separated) types. Bistatic HF radar possesses some inherent advantages over the monostatic case: (1) bistatic HF radar can improve the detection capability of stealth targets; (2) bistatic HF radar can address the ambiguous problem of sea state information extraction; (3) bistatic HF radar can suppress mutual interference between the transmitting and receiving antennas. Therefore, bistatic HF radar has attracted increasing attention internationally. For example, based on the ocean surface electromagnetic scattering theory presented by Walsh [14], Gill et al. [15] developed the first- and second-order ocean surface scattering cross sections for bistatic HF radar, which provides an important theoretical basis for the applications of bistatic HF radar. Subsequently, they investigated the effect of bistatic angle on radar 
cross sections (RCSs) in detail [16], which is helpful to determine a suitable geometry for the deployment of bistatic HF radar. Based on the characteristics of sea clutter for bistatic HF radar, Lipa et al. [17] extracted ocean surface current. Trizna [18] successfully implemented an experiment to map ocean surface current and track the ship target using bistatic HF radar. Grosdidier et al. [19] validated the simulated bistatic HF radar Doppler spectra with the experimental data. Based on the RCS model developed by Gill et al. [15], Huang et al. [20] successfully obtained the unambiguous wind direction on the Southern China coast and the directional ocean wave spectra are respectively extracted from simulated noisy bistatic HF radar data [21] and synthetic bistatic HF radar data [22]. As the application of onshore bistatic HF radar matures, the floating platform based bistatic HF radar (the transmitter is deployed on a floating ocean platform and the receiver is installed on shore) gradually becomes a deployment trend. However, the platform motion may have an important effect on the application of bistatic HF radars.

In the floating platform based monostatic HF radar experiment, some researchers have observed that the platform motion can be viewed as phase modulation of radar Doppler spectra [23-26]. Theoretically, Walsh et al. $[27,28]$ developed the first- and second-order ocean surface scattering cross section models for the case of a transmitter being installed on a floating platform with sway. They pointed out sway can induce additional peaks in radar Doppler spectra. Subsequently, Sun et al. [29] and Ma et al. [30,31] derived corresponding RCSs for shipborne and bistatic cases, respectively. However, based on the seakeeping theory [32,33], the deep-water floating platform generally has six degree-of-freedom (DOF) oscillation motion with multi-frequency due to the interaction between the complex ocean environment and floating platform. More recently, Ma et al. [34] extended the floating platform based bistatic RCSs to a dual-frequency platform motion case incorporating sway and surge. They presented that more additional peaks caused by the combined motion will symmetrically appear in radar Doppler spectra. Yao et al. [35,36] extended the first-order shipborne and bistatic RCSs to a horizontal oscillation motion case with a single-frequency and pointed out yaw may have a more important effect on radar Doppler spectra. Therefore, only considering two-dimensional platform motion with a dual-frequency model for the floating platform based bistatic HF radar is not realistic in practice. In this paper, on the basis of previous works $[30,31,34,36]$, the first- and second-order ocean surface scattering cross sections for the floating platform based bistatic HF radar incorporating a more realistic multi-frequency six DOF oscillation motion model are presented. The results may have significant implications in future investigations for the application of floating-based bistatic HF radars.

The rest of this paper is organized as follows. In Section 2, a multi-frequency six DOF oscillation motion physical model is first developed. Subsequently, the first- and second-order ocean surface scattering cross sections for the floating platform based bistatic HF radar incorporating a single-frequency six DOF oscillation motion model are derived and, then, the results are extended to the multi-frequency case. Section 3 presents the simulation results and comparative analyses with different oscillation motion models and bistatic angles. Section 4 discusses the effect of six DOF oscillation motion and bistatic angle on the application of the floating-based bistatic HF radar in moving target detection and ocean surface dynamics parameter estimation. Conclusions are provided in Section 5.

\section{Derivation}

\subsection{Physical Model}

Due to the interaction between the ocean floating platform and complex ocean environment, based on the seakeeping theory of deep-water floating platform, the motion of the ocean floating platform can be viewed as the superposition of sway, surge, heave, yaw, pitch, and roll with a multi-frequency model $[32,33]$. Figure 1 shows the diagram of six DOF motion for a transmitting sensor on a floating platform. It is assumed that the source is at $(a, b, h)$. According to the work of Walsh et al. in [27], the motion components in vertical direction will not result in additional Doppler effect. Thus, heave will not be considered in the physical model as well as the components of pitch and roll in vertical direction. 
The displacement vectors in horizontal direction caused by sway, surge, yaw, pitch, and roll can be respectively expressed as

$$
\begin{gathered}
\delta \vec{\rho}_{01}(t)=\sum_{j=1}^{N_{1}} a_{1, j} \sin \left(\omega_{1, j}+\phi_{1, j}\right) \delta \hat{\rho}_{01}, \\
\delta \vec{\rho}_{02}(t)=\sum_{j=1}^{N_{2}} a_{2, j} \sin \left(\omega_{2, j}+\phi_{2, j}\right) \delta \hat{\rho}_{02} \\
\delta \vec{\rho}_{03}(t)=2 l_{3} \sin \left[\frac{\theta_{3}(t)}{2}\right] \delta \hat{\rho}_{03}(t) \\
\delta \vec{\rho}_{04}(t)=2 l_{4} \sin \left[\frac{\theta_{4}(t)}{2}\right] \sin \left[\frac{\pi}{2}+\frac{\theta_{4}(t)}{2}-\alpha_{4}\right] \delta \hat{\rho}_{04}, \\
\delta \vec{\rho}_{05}(t)=2 l_{5} \sin \left[\frac{\theta_{5}(t)}{2}\right] \sin \left[\frac{\pi}{2}+\frac{\theta_{5}(t)}{2}-\alpha_{5}\right] \delta \hat{\rho}_{05},
\end{gathered}
$$

where $a_{1, j}$ and $a_{2, j}, \omega_{1, j}$ and $\omega_{2, j}$, and $\phi_{1, j}$ and $\phi_{2, j}$ are the amplitudes, angular frequencies, and initial phases for each frequency component of sway and surge, respectively. $l_{3}=\sqrt{a^{2}+b^{2}}, l_{4}=\sqrt{a^{2}+h^{2}}$, $l_{5}=\sqrt{b^{2}+h^{2}}, \alpha_{4}=\arctan (a / h), \alpha_{5}=\arctan (b / h) . \theta_{3}(t), \theta_{4}(t)$, and $\theta_{5}(t)$ are the rotation angles of yaw, pitch, and roll, respectively, which can be written as

$$
\begin{aligned}
& \theta_{3}(t)=\sum_{j=1}^{N_{3}} \theta_{m 3, j} \sin \left(\omega_{3, j} t+\phi_{3, j}\right), \\
& \theta_{4}(t)=\sum_{j=1}^{N_{4}} \theta_{m 4, j} \sin \left(\omega_{4, j} t+\phi_{4, j}\right), \\
& \theta_{5}(t)=\sum_{j=1}^{N_{5}} \theta_{m 5, j} \sin \left(\omega_{5, j} t+\phi_{5, j}\right),
\end{aligned}
$$

in which $\theta_{m 3, j}, \theta_{m 4, j}$ and $\theta_{m 5, j}, \omega_{3, j}, \omega_{4, j}$ and $\omega_{5, j}$, and $\phi_{3, j}, \phi_{4, j}$ and $\phi_{5, j}$ are the amplitudes, angular frequencies, and initial phases of yaw, pitch, and roll, respectively. $j=1,2, \ldots, N_{i}(i=1,2, \ldots, 5)$ indicates the number of frequency components associated with sway, surge, yaw, pitch, and roll, respectively. $\delta \hat{\rho}_{01}(t), \delta \hat{\rho}_{02}(t), \delta \hat{\rho}_{03}(t), \delta \hat{\rho}_{04}(t)$, and $\delta \hat{\rho}_{05}(t)$ are the corresponding motion directions, respectively, which can be represented by angles $\theta_{01}(t), \theta_{02}(t), \theta_{03}(t), \theta_{04}(t)$, and $\theta_{05}(t)$.

Therefore, the overall displacement vector caused by six DOF oscillation motion with a multi-frequency model can be expressed as

$$
\delta \vec{\rho}_{0}(t)=\delta \vec{\rho}_{01}(t)+\delta \vec{\rho}_{02}(t)+\delta \vec{\rho}_{03}(t)+\delta \vec{\rho}_{04}(t)+\delta \vec{\rho}_{05}(t)
$$

\subsection{RCS Incorporating a Single-Frequency Six DOF Motion Model}

In order to simplify the derivation, a single-frequency six DOF oscillation motion model is first considered. That is, $N_{i}=1(i=1,2, \ldots, 5)$ in Equation (9). 


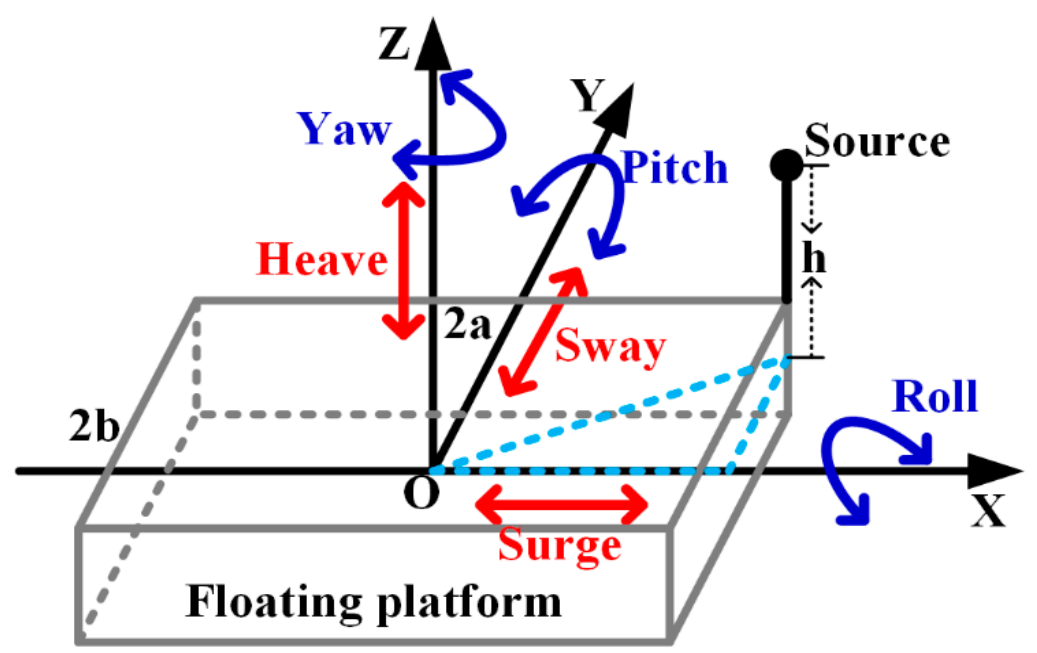

Figure 1. Diagram of six degree-of-freedom (DOF) motion of floating platform.

\subsubsection{First-Order RCS}

Figure 2 shows the first-order bistatic HF radar scatter geometry for the case of the source being installed on an ocean floating platform. In [30], Ma et al. derived the first-order bistatic HF RCS when the source is deployed on a floating platform with a single-frequency sway motion. Then, different platform motion models are introduced to derive corresponding RCS [29,34-36]. In this study, based on the ocean surface electromagnetic scattering theory, a more realistic six DOF oscillation motion model is established and the first-order bistatic HF RCS model can be modified to

$$
\begin{gathered}
\sigma_{1}\left(\omega_{d}\right)=2^{2} k_{0}^{2} \Delta \rho \sum_{m= \pm 1} \int_{K} K^{2} \cos \phi_{0} S_{1}(m \vec{K}) S a^{2}\left[\frac{\Delta \rho}{2}\left(\frac{K}{\cos \phi_{0}}-2 k_{0}\right)\right] \\
\cdot \int_{\tau} e^{-j \tau\left(m \sqrt{g K}+\omega_{d}\right)}\left\langle M\left(K, \theta_{K^{\prime}}, \tau, t\right)\right\rangle d \tau d K
\end{gathered},
$$

where $\omega_{d}$ is the Doppler frequency, $k_{0}$ is the radian wavenumber, $\Delta \rho$ is the patch width, $\vec{K}=\left(K, \theta_{\vec{K}}\right)$ is the ocean wave vector, $S_{1}(\cdot)$ indicates the directional ocean wave spectrum, $S a(\cdot)$ represents the sinc function, $\tau$ is the interval between samples, $g$ is the gravitational acceleration and

$$
\begin{aligned}
M\left(K, \theta_{\vec{K}^{\prime}} \tau, t\right) & =e^{-\frac{j}{2}\left\{K \delta \rho_{0}(t) \cos \left[\theta_{\vec{K}}-\theta_{0}(t)\right]\right\}} e^{\frac{j}{2}\left\{K \delta \rho_{0}(t+\tau) \sin \left[\theta_{\vec{K}}-\theta_{0}(t+\tau)\right]\right\}} \\
& \cdot e^{-\frac{j \tan \phi_{0}}{2}\left\{K \delta \rho_{0}(t) \sin \left[\theta_{\vec{K}}-\theta_{0}(t)\right]\right\}} e^{\frac{j \tan \phi_{0}}{2}\left\{K \delta \rho_{0}(t+\tau) \sin \left[\theta_{\vec{K}}-\theta_{0}(t+\tau)\right]\right\}}
\end{aligned} .
$$

By substituting the displacement term in Equation (9) into Equation (11), the ensemble average of $M\left(K, \theta_{K^{\prime}}, \tau, t\right)$ can be derived as

$$
\left\langle M\left(K, \theta_{\vec{K}^{\prime}}, \tau, t\right)\right\rangle=\left\langle M_{1} M_{2} M_{3} M_{4} M_{5}\right\rangle
$$

where

$$
\begin{gathered}
M_{1}=e^{j V_{1} \cos Q_{1},} \\
M_{2}=e^{j V_{2} \cos Q_{2}}, \\
M_{3}=e^{j V_{31} \cos \left(2 Q_{3}\right)} e^{j V_{32} \cos Q_{3}}, \\
M_{4}=e^{j V_{41} \sin \left(2 Q_{4}\right)} e^{j V_{42} \cos Q_{4}},
\end{gathered}
$$

and

$$
M_{5}=e^{j V_{51} \sin \left(2 Q_{5}\right)} e^{j V_{52} \cos Q_{5}}
$$


in which

$$
\begin{gathered}
V_{1}=a_{1} K\left[\cos \left(\theta_{\vec{K}}-\theta_{01}\right)+\tan \phi_{0} \sin \left(\theta_{\vec{K}}-\theta_{01}\right)\right] \sin \frac{\omega_{1} \tau}{2}, \\
V_{2}=a_{2} K\left[\cos \left(\theta_{\vec{K}}-\theta_{02}\right)+\tan \phi_{0} \sin \left(\theta_{\vec{K}}-\theta_{02}\right)\right] \sin \frac{\omega_{2} \tau}{2}, \\
V_{31}=-\frac{K l_{3} \theta_{m 3}^{2} \cos \left(\theta_{\vec{K}}-\theta^{\prime}\right)}{4}\left(1+\tan \phi_{0}\right) \sin \omega_{3} \tau, \\
V_{32}=-K l_{3} \theta_{m 3} \cos \left(\theta_{\vec{K}}-\theta^{\prime}\right)\left(-1+\tan \phi_{0}\right) \sin \frac{\omega_{3} \tau}{2}, \\
V_{41}=\frac{K l_{4} \theta_{m 4}^{2} \cos \left(\theta_{\vec{K}}-\theta_{04}\right) \sin \alpha_{4}}{2}\left(1+\frac{\tan \phi_{0}}{2}\right) \sin \omega_{4} \tau, \\
V_{42}=2 K l_{4} \theta_{m 4} \cos \left(\theta_{\vec{K}}-\theta_{04}\right) \cos \alpha_{4}\left(1+\frac{\tan \phi_{0}}{2}\right) \sin \frac{\omega_{4} \tau}{2}, \\
V_{51}=\frac{K l_{5} \theta_{m 5}^{2} \cos \left(\theta_{\vec{K}}-\theta_{05}\right) \sin \alpha_{5}}{2}\left(1+\frac{\tan \phi_{0}}{2}\right) \sin \omega_{5} \tau, \\
V_{52}=2 K l_{5} \theta_{m 5} \cos \left(\theta_{\vec{K}}-\theta_{05}\right) \cos \alpha_{5}\left(1+\frac{\tan \phi_{0}}{2}\right) \sin \frac{\omega_{5} \tau}{2}, \\
Q_{i}=\omega_{i} t+\frac{\omega_{i} \tau}{2}+\phi_{i}(i=1,2, \ldots, 5)
\end{gathered}
$$

and $\theta^{\prime}=\arctan (b / a)$.

Using the Euler equation and the property of the Bessel function

$$
\begin{gathered}
e^{j x}=\cos x+j \sin x \\
\cos (V \sin Q)=J_{0}(V)+2 \sum_{n=1}^{+\infty} J_{2 n}(V) \cos (2 n Q) \\
\sin (V \sin Q)=2 \sum_{n=1}^{+\infty} J_{2 n-1}(V) \cos [(2 n-1) Q] \\
\cos (V \cos Q)=J_{0}(V)+2 \sum_{n=1}^{+\infty}(-1)^{n} J_{2 n}(V) \cos (2 n Q)
\end{gathered}
$$

and

$$
\sin (V \cos Q)=-2 \sum_{n=1}^{+\infty}(-1)^{n} J_{2 n-1}(V) \cos [(2 n-1) Q],
$$

where $J_{n}$ is the $n$-th order Bessel function. Then, Equation (12) can be reduced to

$$
\left\langle M\left(K, \theta_{\bar{K}^{\prime}} \tau, t\right)\right\rangle=J_{0}\left(V_{1}\right) J_{0}\left(V_{2}\right) J_{0}\left(V_{31}\right) J_{0}\left(V_{32}\right) J_{0}\left(V_{41}\right) J_{0}\left(V_{42}\right) J_{0}\left(V_{51}\right) J_{0}\left(V_{52}\right) .
$$

By taking advantage of the relationship of Bessel function

$$
\begin{gathered}
J_{0}\left(2 x \sin \frac{\Phi}{2}\right)=\sum_{n=-\infty}^{+\infty} J_{n}^{2}(x) \cos (n \Phi), \\
J_{n}(-x)=(-1)^{n} J_{n}(x),
\end{gathered}
$$


and similar derivation in [34], Equation (32) can be further modified to

$$
\begin{aligned}
\left\langle M\left(K, \theta_{\mathrm{K}^{\prime}} \tau, t\right)\right\rangle= & \sum_{n_{1}=-\infty}^{+\infty} J_{n_{1}}^{2}\left(X_{1}\right) \cos \left(n_{1} \omega_{1} \tau\right) \sum_{n_{2}=-\infty}^{+\infty} J_{n_{2}}^{2}\left(X_{2}\right) \cos \left(n_{2} \omega_{2} \tau\right) \\
& \cdot \sum_{n_{31}=-\infty}^{+\infty} J_{n_{31}}^{2}\left(X_{31}\right) \cos \left(2 n_{31} \omega_{3} \tau\right) \sum_{n_{32}=-\infty}^{+\infty} J_{n_{32}}^{2}\left(X_{32}\right) \cos \left(n_{32} \omega_{3} \tau\right) \\
& \cdot \sum_{n_{41}=-\infty}^{+\infty} J_{n_{41}}^{2}\left(X_{41}\right) \cos \left(2 n_{41} \omega_{4} \tau\right) \sum_{n_{42}=-\infty}^{+\infty} J_{n_{42}}^{2}\left(X_{42}\right) \cos \left(n_{42} \omega_{4} \tau\right) \\
& \cdot \sum_{n_{51}=-\infty}^{+\infty} J_{n_{51}}^{2}\left(X_{51}\right) \cos \left(2 n_{51} \omega_{5} \tau\right) \sum_{n_{52}=-\infty}^{+\infty} J_{n_{52}}^{2}\left(X_{52}\right) \cos \left(n_{52} \omega_{5} \tau\right)
\end{aligned}
$$

where

$$
\begin{gathered}
X_{1}=\frac{a_{1} K\left[\cos \left(\theta_{\vec{K}}-\theta_{01}\right)+\tan \phi_{0} \sin \left(\theta_{\vec{K}}-\theta_{01}\right)\right]}{2}, \\
X_{2}=\frac{a_{2} K\left[\cos \left(\theta_{\vec{K}}-\theta_{02}\right)+\tan \phi_{0} \sin \left(\theta_{\vec{K}}-\theta_{02}\right)\right]}{2}, \\
X_{31}=-\frac{K l_{3} \theta_{m 3}^{2} \cos \left(\theta_{\vec{K}}-\theta^{\prime}\right)}{8}\left(1+\tan \phi_{0}\right) \\
X_{32}=-\frac{K l_{3} \theta_{m 3} \cos \left(\theta_{\vec{K}}-\theta^{\prime}\right)}{2}\left(-1+\tan \phi_{0}\right) \\
X_{41}=\frac{K l_{4} \theta_{m 4}^{2} \cos \left(\theta_{\vec{K}}-\theta_{04}\right) \sin \alpha_{4}}{4}\left(1+\frac{\tan \phi_{0}}{2}\right) \\
X_{42}=K l_{4} \theta_{m 4} \cos \left(\theta_{\vec{K}}-\theta_{04}\right) \cos \alpha_{4}\left(1+\frac{\tan \phi_{0}}{2}\right) \\
X_{51}=\frac{K l_{5} \theta_{m 5}^{2} \cos \left(\theta_{\vec{K}}-\theta_{05}\right) \sin \alpha_{5}}{4}\left(1+\frac{\tan \phi_{0}}{2}\right) \\
X_{52}=K l_{5} \theta_{m 5} \cos \left(\theta_{\vec{K}}-\theta_{05}\right) \cos \alpha_{5}\left(1+\frac{\tan \phi_{0}}{2}\right)
\end{gathered}
$$

Substituting Equation (35) into Equation (10), using the relationship $\cos x=\frac{e^{j x}+e^{-j x}}{2}$ and then completing $\tau$ integration, the first-order ocean surface scattering cross section for bistatic HF radar incorporating a single-frequency six DOF oscillation motion model can be finally derived as

$$
\begin{aligned}
\sigma_{1}\left(\omega_{d}\right)= & 2^{3} \pi k_{0}^{2} \Delta \rho \sum_{m= \pm 1} \int_{K} K^{2} \cos \phi_{0} S_{1}(m \vec{K}) S a^{2}\left[\frac{\Delta \rho}{2}\left(\frac{K}{\cos \phi_{0}}-2 k_{0}\right)\right] \\
& \cdot \sum_{n_{1}=-\infty}^{+\infty} J_{n_{1}}^{2}\left(X_{1}\right) \sum_{n_{2}=-\infty}^{+\infty} J_{n_{2}}^{2}\left(X_{2}\right) \sum_{n_{31}=-\infty}^{+\infty} J_{n_{31}}^{2}\left(X_{31}\right) \sum_{n_{32}=-\infty}^{+\infty} J_{n_{32}}^{2}\left(X_{32}\right) \\
& \cdot \sum_{n_{41}=-\infty}^{+\infty} J_{n_{41}}^{2}\left(X_{41}\right) \sum_{n_{42}=-\infty}^{+\infty} J_{n_{42}}^{2}\left(X_{42}\right) \sum_{n_{51}=-\infty}^{+\infty} J_{n_{51}}^{2}\left(X_{51}\right) \sum_{n_{52}=-\infty}^{+\infty} J_{n_{52}}^{2}\left(X_{52}\right) \\
& \cdot \delta\left(\omega_{d}+m \sqrt{g K}-\sum_{i=1}^{2} n_{i} \omega_{i}-\sum_{i=3}^{5}\left(2 n_{i 1}+n_{i 2}\right) \omega_{i}\right) d K
\end{aligned}
$$




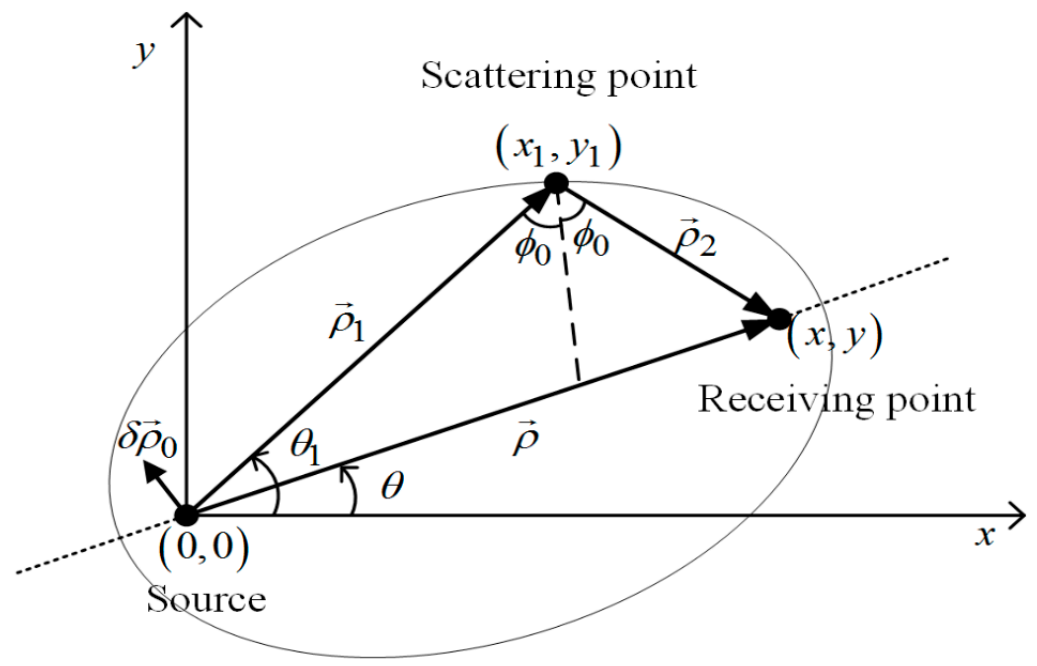

Figure 2. First-order bistatic high-frequency $(\mathrm{HF})$ radar scatter geometry with antenna motion. $(0,0)$, $\left(x_{1}, y_{1}\right)$, and $(x, y)$ represent the source, the scattering point, and the receiving point, respectively. $\delta \vec{\rho}_{0}$ is the displacement vector caused by the source motion. $\theta$ is the angle between the $x$-axis and the direction of the receiving point. $\theta_{1}$ is the angle between the $x$-axis and the direction of the scattering point. $\vec{\rho}$ is the displacement vector from the source to the receiving point. $\vec{\rho}_{1}$ is the displacement vector from the source to the scattering point. $\vec{\rho}_{2}$ is the displacement vector from the scattering point to the receiving point and $\phi_{0}$ indicates the bistatic angle.

\subsubsection{Second-Order RCS}

In general, the second-order RCS is mainly composed of two parts. One is due to single ocean surface scatter from a second-order ocean wave and the scatter geometry is similar to Figure 2. Its difference from the first-order RCS is that the first-order ocean wave at the scattering point is replaced by a second-order ocean wave. The other is due to double scatters from two first-order ocean waves and the scatter geometry is shown in Figure 3. In [31], Ma et al. derived the second-order bistatic HF RCS when the source is deployed on a floating platform with a single-frequency sway motion. Then, different platform motion models are introduced to derive corresponding second-order RCS [29,34]. In this study, based on the scattering theory in [31], a more realistic six DOF oscillation motion model is established and the second-order bistatic HF RCS model can be modified to

$$
\begin{aligned}
\sigma_{2}\left(\omega_{d}\right)= & 2^{2} k_{0}^{2} \Delta \rho \sum_{m_{1}= \pm 1} \sum_{m_{2}= \pm 1} \int_{K} \int_{\theta_{\vec{K}_{1}}} \int_{K_{1}} S_{1}\left(m_{1} \vec{K}_{1}\right) S_{1}\left(m_{2} \vec{K}_{2}\right) \\
& \cdot|\Gamma|^{2} K^{2} K_{1} \cos \phi_{0} S a^{2}\left[\frac{\Delta \rho}{2}\left(\frac{K}{\cos \phi_{0}}-2 k_{0}\right)\right] \\
& \cdot \int_{\tau} e^{-j \tau\left(m_{1} \sqrt{g K_{1}}++m_{2} \sqrt{g K_{2}}+\omega_{d}\right)}\left\langle M\left(K, \theta_{\vec{K}^{\prime}} \tau, t\right)\right) d \tau d K_{1} d \theta_{\vec{K}_{1}} d K
\end{aligned}
$$

where $\vec{K}=\vec{K}_{1}+\vec{K}_{2}, \vec{K}_{1}=\left(K_{1}, \theta_{\vec{K}_{1}}\right)$, and $\vec{K}_{2}=\left(K_{2}, \theta_{\vec{K}_{2}}\right)$ indicate the two first-order ocean waves, respectively. $|\Gamma|=\left|\Gamma_{H}\right|+\left|\Gamma_{E}\right|$ represents the total coupling coefficient, $\left|\Gamma_{H}\right|$ is the hydrodynamic coupling coefficient of two first-order ocean waves [37] and $\left|\Gamma_{E}\right|$ is the electromagnetic coupling coefficient [28]. 


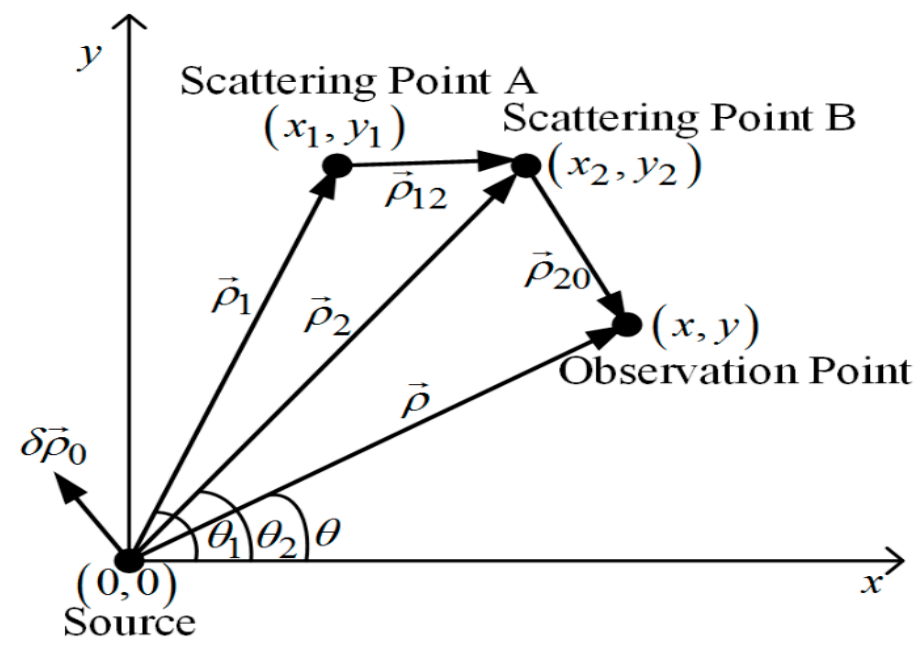

Figure 3. Second-order bistatic HF radar scatter geometry with antenna motion for the case of a double scatter from two first-order ocean waves. $(0,0),\left(x_{1}, y_{1}\right),\left(x_{2}, y_{2}\right)$, and $(x, y)$ represent the source, the first scattering point $\mathrm{A}$, the second scattering point $\mathrm{B}$, and the receiving point, respectively. $\theta$ is the angle between the $x$-axis and the direction of the receiving point. $\theta_{1}$ is the angle between $x$-axis and the direction of the scattering point A. $\theta_{2}$ is the angle between $x$-axis and the direction of the scattering point B. $\delta \vec{\rho}_{0}$ is the displacement vector caused by the motion of the source. $\vec{\rho}$ is the displacement vector from the source to the receiving point. $\vec{\rho}_{1}$ is the displacement vector from the source to the scattering point A. $\vec{\rho}_{2}$ is the displacement vector from the source to the scattering point $B . \vec{\rho}_{12}$ is the displacement vector from the scattering point A to the scattering point B. $\vec{\rho}_{20}$ is the displacement vector from the scattering point $B$ to the observation point.

Substituting a single-frequency six DOF oscillation motion model (Equation (9)) into the second-order bistatic HF RCS model (Equation (45)), similar to the analysis of the first-order RCS in Section 2.2.1, the second-order ocean surface scattering cross section for bistatic HF radar can be finally derived as

$$
\begin{aligned}
\sigma_{2}\left(\omega_{d}\right)= & 2^{3} \pi k_{0}^{2} \Delta \rho \sum_{m_{1}= \pm 1} \sum_{m_{2}= \pm 1} \int_{K} \int_{\theta_{\vec{K}_{1}}} \int_{K_{1}} S_{1}\left(m_{1} \vec{K}_{1}\right) S_{1}\left(m_{2} \vec{K}_{2}\right) \\
& \cdot|\Gamma|^{2} K^{2} K_{1} \cos \phi_{0} S a^{2}\left[\frac{\Delta \rho}{2}\left(\frac{K}{\cos \phi_{0}}-2 k_{0}\right)\right] \\
& \cdot \sum_{n_{1}=-\infty}^{+\infty} J_{n_{1}}^{2}\left(X_{1}\right) \sum_{n_{2}=-\infty}^{+\infty} J_{n_{2}}^{2}\left(X_{2}\right) \sum_{n_{31}=-\infty}^{+\infty} J_{n_{31}}^{2}\left(X_{31}\right) \sum_{n_{32}=-\infty}^{+\infty} J_{n_{32}}^{2}\left(X_{32}\right) \\
& \cdot \sum_{n_{41}=-\infty}^{+\infty} J_{n_{41}}^{2}\left(X_{41}\right) \sum_{n_{42}=-\infty}^{+\infty} J_{n_{42}}^{2}\left(X_{42}\right) \sum_{n_{51}=-\infty}^{+\infty} J_{n_{51}}^{2}\left(X_{51}\right) \sum_{n_{52}=-\infty}^{+\infty} J_{n_{52}}^{2}\left(X_{52}\right) \\
& \cdot \delta\left(\omega_{d}++m_{1} \sqrt{g K_{1}}+m_{2} \sqrt{g K_{2}}-\sum_{i=1}^{2} n_{i} \omega_{i}-\sum_{i=3}^{5}\left(2 n_{i 1}+n_{i 2}\right) \omega_{i}\right) d K_{1} d \theta_{K_{1}} d K
\end{aligned}
$$

\subsection{RCS Incorporating a Multi-Frequency Six DOF Motion Model}

In this section, a general six DOF oscillation motion model incorporating multi-frequency components is considered. Combing the derivation in Section 2.2 and the analysis in [34], the 
first- and second-order ocean surface scattering cross sections for bistatic HF radar incorporating a multi-frequency six DOF oscillation motion model can be respectively expressed as

$$
\begin{aligned}
\sigma_{1}\left(\omega_{d}\right) & =2^{3} \pi k_{0}^{2} \Delta \rho \sum_{m= \pm 1} J_{K} K^{2} \cos \phi_{0} S_{1}(m \vec{K}) S a^{2}\left[\frac{\Delta \rho}{2}\left(\frac{K}{\cos \phi_{0}}-2 k_{0}\right)\right] \\
& \cdot \sum_{j=1}^{N_{1}}\left[\sum_{n_{1, j}=-\infty}^{+\infty} J_{n_{1, j}}^{2}\left(X_{1, j}\right)\right] \sum_{j=1}^{N_{2}}\left[\sum_{n_{2, j}=-\infty}^{+\infty} J_{n_{2, j}}^{2}\left(X_{2, j}\right)\right] \\
& \cdot \sum_{j=1}^{N_{3}}\left[\sum_{n_{31, j}=-\infty}^{+\infty} J_{n_{31, j}}^{2}\left(X_{31, j}\right) \sum_{n_{32, j}=-\infty}^{+\infty} J_{n_{32, j}}^{2}\left(X_{32, j}\right)\right] \\
& \cdot \sum_{j=1}^{N_{4}}\left[\sum_{n_{41, j}=-\infty}^{+\infty} J_{n_{41, j}}^{2}\left(X_{41, j}\right) \sum_{n_{42, j}=-\infty}^{+\infty} J_{n_{42, j}}^{2}\left(X_{42, j}\right)\right] \\
& \cdot \sum_{j=1}^{N_{5}}\left[\sum_{n_{51, j}=-\infty}^{+\infty} J_{n_{51, j}}^{2}\left(X_{51, j}\right) \sum_{n_{52, j}=-\infty}^{+\infty} J_{n_{52, j}}^{2}\left(X_{52, j}\right)\right] \\
& \cdot \delta\left\{\omega_{d}+m \sqrt{g K}-\sum_{i=1}^{2}\left[\sum_{j=1}^{N_{i}} n_{i, j} \omega_{i, j}\right]-\sum_{i=3}^{5}\left[\sum_{j=1}^{N_{i}}\left(2 n_{i 1, j}+n_{i 2, j}\right) \omega_{i, j}\right]\right\} d K
\end{aligned}
$$

and

$$
\begin{aligned}
& \sigma_{2}\left(\omega_{d}\right)=2^{3} \pi k_{0}^{2} \Delta \rho \sum_{m_{1}= \pm 1} \sum_{m_{2}= \pm 1} \int_{K} \int_{\theta_{\vec{K}_{1}}} \int_{K_{1}} S_{1}\left(m_{1} \vec{K}_{1}\right) S_{1}\left(m_{2} \vec{K}_{2}\right) \\
& \cdot|\Gamma|^{2} K^{2} K_{1} \cos \phi_{0} S a^{2}\left[\frac{\Delta \rho}{2}\left(\frac{K}{\cos \phi_{0}}-2 k_{0}\right)\right] \\
& \cdot \sum_{j=1}^{N_{1}}\left[\sum_{n_{1, j}=-\infty}^{+\infty} J_{n_{1, j}}^{2}\left(X_{1, j}\right)\right] \sum_{j=1}^{N_{2}}\left[\sum_{n_{2, j}=-\infty}^{+\infty} J_{n_{2, j}}^{2}\left(X_{2, j}\right)\right] \\
& \cdot \sum_{j=1}^{N_{3}}\left[\sum_{n_{31, j}=-\infty}^{+\infty} J_{n_{31, j}}^{2}\left(X_{31, j}\right) \sum_{n_{32, j}=-\infty}^{+\infty} J_{n_{32, j}}^{2}\left(X_{32, j}\right)\right] \\
& \cdot \sum_{j=1}^{N_{4}}\left[\sum_{n_{41, j}=-\infty}^{+\infty} J_{n_{41, j}}^{2}\left(X_{41, j}\right) \sum_{n_{42, j}=-\infty}^{+\infty} J_{n_{42, j}}^{2}\left(X_{42, j}\right)\right] \\
& \cdot \sum_{j=1}^{N_{5}}\left[\sum_{n_{51, j}=-\infty}^{+\infty} J_{n_{51, j}}^{2}\left(X_{51, j}\right) \sum_{n_{52, j}=-\infty}^{+\infty} J_{n_{52, j}}^{2}\left(X_{52, j}\right)\right] \\
& \cdot \delta\left\{\omega_{d}+m_{1} \sqrt{g K_{1}}+m_{2} \sqrt{g K_{2}}-\sum_{i=1}^{2}\left[\sum_{j=1}^{N_{i}} n_{i, j} \omega_{i, j}\right]-\sum_{i=3}^{5}\left[\sum_{j=1}^{N_{i}}\left(2 n_{i 1, j}+n_{i 2, j}\right) \omega_{i, j}\right]\right\} \\
& \cdot d K_{1} d \theta_{K_{1}} d K
\end{aligned}
$$

where

$$
\begin{gathered}
X_{1, j}=\frac{a_{1, j} K\left[\cos \left(\theta_{\vec{K}}-\theta_{01}\right)+\tan \phi_{0} \sin \left(\theta_{\vec{K}}-\theta_{01}\right)\right]}{2}, \\
X_{2, j}=\frac{a_{2, j} K\left[\cos \left(\theta_{\vec{K}}-\theta_{02}\right)+\tan \phi_{0} \sin \left(\theta_{\vec{K}}-\theta_{02}\right)\right]}{2}, \\
X_{31, j}=-\frac{K l_{3} \theta_{m 3, j}^{2} \cos \left(\theta_{\vec{K}}-\theta^{\prime}\right)}{8}\left(1+\tan \phi_{0}\right), \\
X_{32, j}=-\frac{K l_{3} \theta_{m 3, j} \cos \left(\theta_{\vec{K}}-\theta^{\prime}\right)}{2}\left(-1+\tan \phi_{0}\right), \\
X_{41, j}=\frac{K l_{4} \theta_{m 4, j}^{2} \cos \left(\theta_{\vec{K}}-\theta_{04}\right) \sin \alpha_{4}}{4}\left(1+\frac{\tan \phi_{0}}{2}\right),
\end{gathered}
$$




$$
\begin{aligned}
& X_{42, j}=K l_{4} \theta_{m 4, j} \cos \left(\theta_{\vec{K}}-\theta_{04}\right) \cos \alpha_{4}\left(1+\frac{\tan \phi_{0}}{2}\right), \\
& X_{51, j}=\frac{K l_{5} \theta_{m 5, j}^{2} \cos \left(\theta_{\vec{K}}-\theta_{05}\right) \sin \alpha_{5}}{4}\left(1+\frac{\tan \phi_{0}}{2}\right), \\
& X_{52, j}=K l_{5} \theta_{m 5, j} \cos \left(\theta_{\vec{K}}-\theta_{05}\right) \cos \alpha_{5}\left(1+\frac{\tan \phi_{0}}{2}\right) .
\end{aligned}
$$

From Equations (47) and (48), it is obvious that the derived RCSs can be reduced to some existing results. For example, if only the sway motion of the floating platform is considered, the derived results can be easily reduced to the Ma et al. results [30,31]. If only the sway and surge motions with a dual-frequency model are considered, the derived results agree with those of Ma et al. [34]. If a horizontal oscillation motion model is considered, the derived first-order RCS is consistent with that derived by Yao et al. [36]. In particular, for the case of a stationary ocean platform, the derived results can be readily reduced to the onshore bistatic case [15]. In addition, it should be noted that the firstand second-order ocean surface scattering cross sections for monostatic HF radar incorporating a multi-frequency six DOF oscillation motion model can be easily derived if the bistatic angle is set to zero in Equations (47) and (48).

\section{Simulation Results}

In this study, using the product of a Pierson-Moskowiz ocean spectral model [38] and a cardioid directional factor [39] as directional ocean wave spectrum, simulations are conducted to analyze the effect of the antenna motion on radar Doppler spectra. The radar operating frequency, range resolution, bistatic angle, and the angle of ellipse normal are set to $5 \mathrm{MHz}, 3 \mathrm{~km}, 45^{\circ}$, and $90^{\circ}$, respectively. The platform parameters are obtained from a deep-water floating platform [40], where the length and width of the platform are, respectively, 240 and $46 \mathrm{~m}$. A single-frequency six DOF oscillation motion for wave frequency (WF) is first considered and the oscillation motion parameters are listed in Table 1. The wind speed and direction are, respectively, $41.12 \mathrm{~m} / \mathrm{s}$ and $180^{\circ}$ with respect to the direction of ellipse normal. A Hamming window is added to smooth the derived RCS curves.

Table 1. Single-frequency six DOF oscillation motion parameters for wave frequency (WF).

\begin{tabular}{cccccc}
\hline Parameters & Sway & Surge & Yaw & Pitch & Roll \\
\hline Frequency (rad/s) & 0.35 & 0.3 & 0.4 & 0.35 & 0.3 \\
Amplitude & $1.192 \mathrm{~m}$ & $1.785 \mathrm{~m}$ & $1.336^{\circ}$ & $1.501^{\circ}$ & $0.631^{\circ}$ \\
\hline
\end{tabular}

\subsection{Comparison with Onshore Case}

Comparisons of the RCSs for floating-based monostatic and bistatic radars with those for onshore monostatic and bistatic cases are shown in Figures 4-6. It should be noted that only sway is considered in this part. From Figure 4a, the locations of the Bragg peaks of the onshore monostatic and bistatic HF radars are $\omega_{m B}= \pm \sqrt{2 g k_{0}}$ and $\omega_{b B}= \pm \sqrt{2 g k_{0} \cos \phi_{0}}$, respectively. It is apparent that the locations of the Bragg peaks of the bistatic HF radar are closer to zero frequency and the amplitudes are lower than those of the monostatic case. This is because a $\cos \phi_{0}$ term exists in Equation (47). From Figure $4 b$, it is seen that sway can result in some additional peaks symmetrically appearing in both monostatic and bistatic RCS curves. The amplitudes of these sway-induced peaks are generally lower than those of the Bragg peaks and the locations are respectively at $\omega_{m B}+n_{1} \omega_{1}$ and $\omega_{b B}+n_{1} \omega_{1}$ for the floating-based monostatic and bistatic cases. However, the locations of the Bragg peaks remain unchanged and the amplitudes are slightly lower than those of onshore cases. 


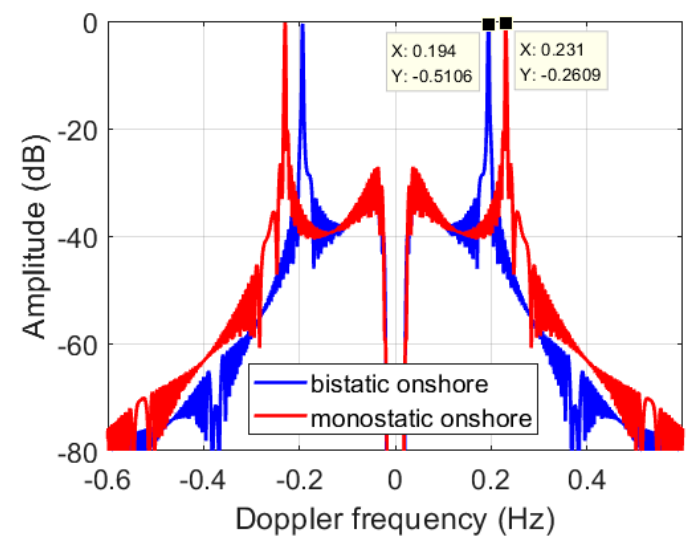

(a)

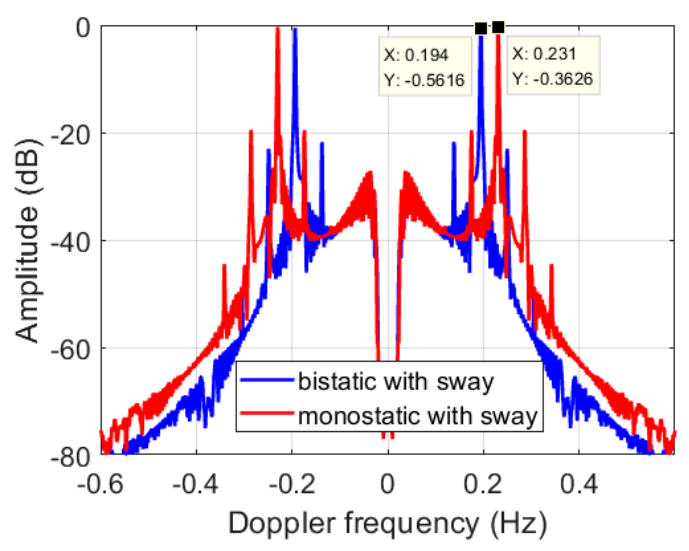

(b)

Figure 4. Simulated first-order radar cross sections (RCSs), (a) for onshore monostatic and bistatic cases; (b) for floating-based monostatic and bistatic cases with sway.

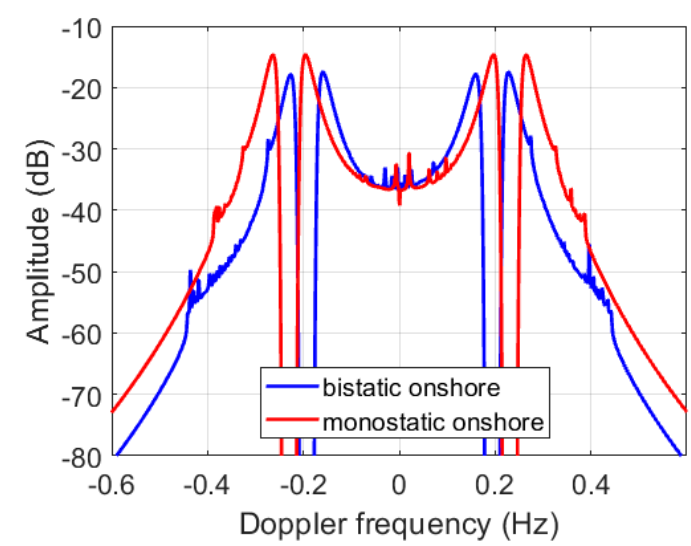

(a)

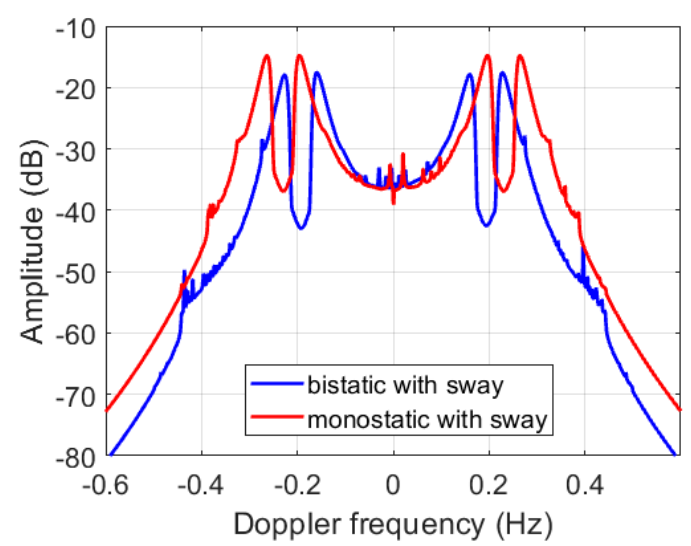

(b)

Figure 5. Simulated second-order RCSs, (a) for onshore monostatic and bistatic cases; (b) for floating-based monostatic and bistatic cases with sway.

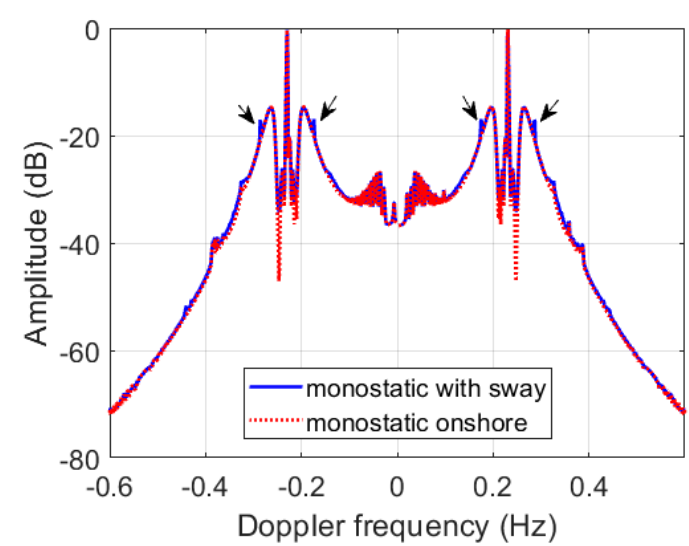

(a)

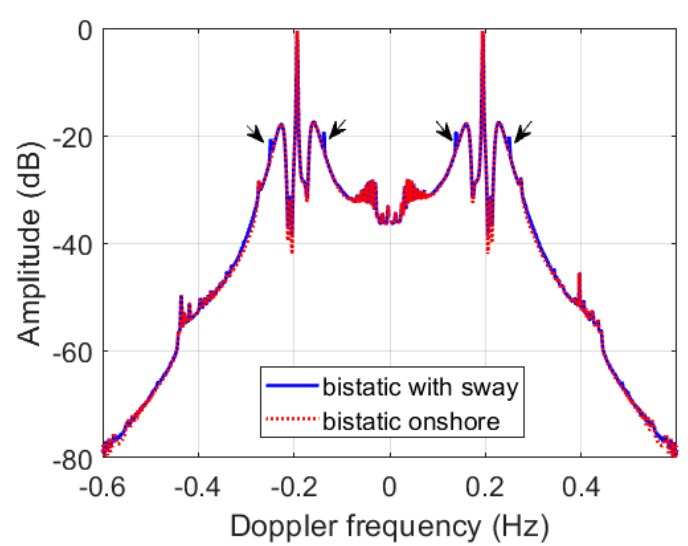

(b)

Figure 6. Simulated total RCSs containing first- and second-order RCSs, (a) for onshore and floating-based monostatic cases; (b) for onshore and floating-based bistatic cases. The black arrows represent additional sway-induced peaks. 
As mentioned before, the second-order RCS mainly contains the hydrodynamic and electromagnetic contributions. For onshore bistatic radar, the locations of the hydrodynamic and electromagnetic peaks are respectively given as [15]

$$
\omega_{h d}= \pm \sqrt{2} \omega_{b B},
$$

and

$$
\omega_{e d}= \pm 2^{3 / 4} \sqrt{\frac{\left(1 \pm \sin \phi_{0}\right)^{1 / 2}}{\cos \phi_{0}}} \omega_{b B} .
$$

For onshore monostatic case, the corresponding locations can be obtained by imposing $\phi_{0}=0$ in Equations (57) and (58). The amplitudes of the second-order RCS for the onshore bistatic case are lower than those of the onshore monostatic case, as shown in Figure 5a. This is because there exists a $\cos \phi_{0}$ term in Equation (48) compared with the monostatic second-order RCS. Theoretically, additional sway-induced peaks will appear in the second-order RCS curves at frequencies $\omega_{h d}+n_{1} \omega_{1}$ and $\omega_{e d}+n_{1} \omega_{1}$ for the floating bistatic case. However, from Figure $5 \mathrm{~b}$, those additional sway-induced peaks are imperceptible. Although the effect of sway on the second-order RCS is not apparent, the additional peaks appearing in the first-order RCS curve may raise the second-order RCS, as shown in Figure 6.

\subsection{Effect of Six DOF Motion on RCS}

Figure 7 shows the simulated first-order RCSs for different platform motion models. It can be seen that each one-dimensional oscillation motion will induce additional peaks. The locations and amplitudes of these additional motion-induced peaks are different from each other, which are decided by the frequency and amplitude of corresponding oscillation motion, respectively. From Equation (47), the initial phase of each one-dimensional oscillation motion has no effect on RCSs. Therefore, the oscillation motion of the floating platform can be regarded as frequency modulation of RCS of the onshore bistatic HF radar. From Figure 7d,e, pitch and roll have a smaller effect on RCSs compared to other oscillation motions. This is because the oscillation amplitudes of pitch and roll are relatively small. However, yaw results in more additional peaks appearing in the first-order RCS curve with a small oscillation amplitude and the amplitudes of these yaw-induced peaks are higher with respect to other cases. This is because the radar antenna is generally installed at the edge of the platform (especially a ship) to reduce the electromagnetic effect of platform superstructures on radar Doppler spectra. In this study, the antenna is assumed to be deployed far from the center of rotation. Thus, a small oscillation amplitude of yaw may cause a large horizontal antenna displacement.

When a six DOF oscillation motion model is considered, more additional motion-induced peaks will appear in the first-order RCS curve, which are not only caused by each one-dimensional oscillation motion but also by the combined motion. For such a case, the frequency locations of these motion-induced peaks can be expressed as

$$
\omega_{d}=\omega_{b B}+\sum_{i=1}^{2} n_{i} \omega_{i}+\sum_{i=3}^{5}\left(2 n_{i 1}+n_{i 2}\right) \omega_{i} .
$$

Therefore, the modulation effect on the first-order RCS of six DOF oscillation motion is significantly greater than that of each one-dimensional oscillation motion. 


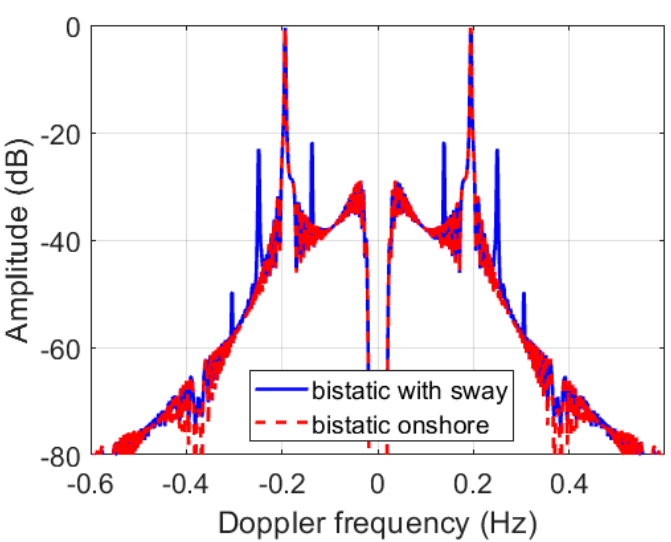

(a)

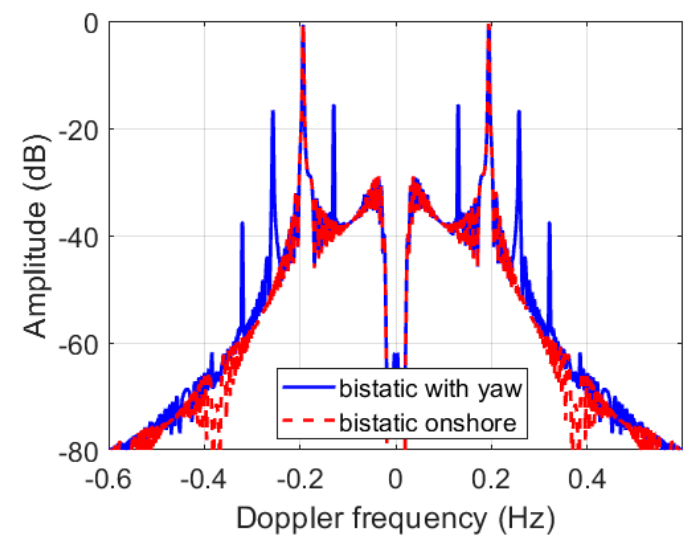

(c)

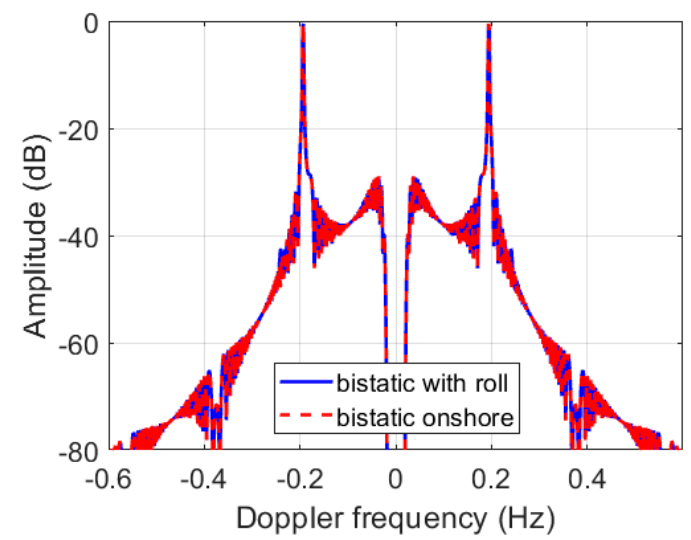

(e)

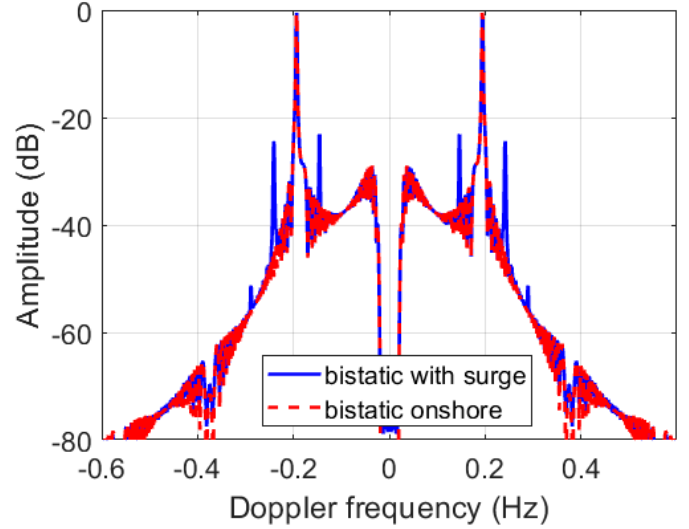

(b)

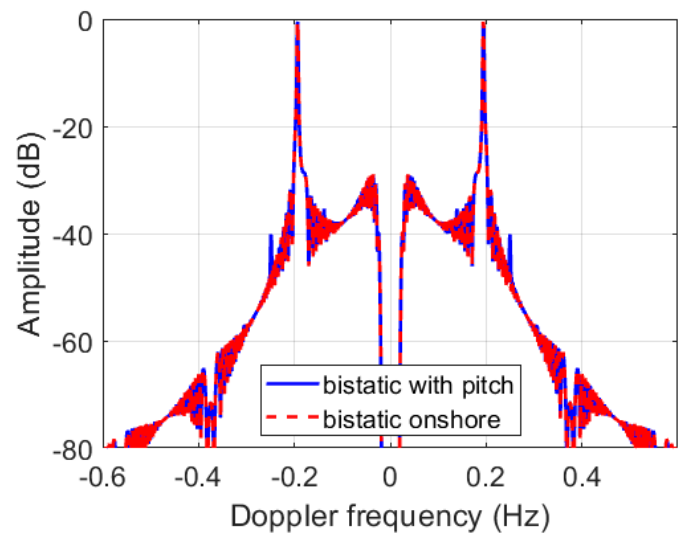

(d)

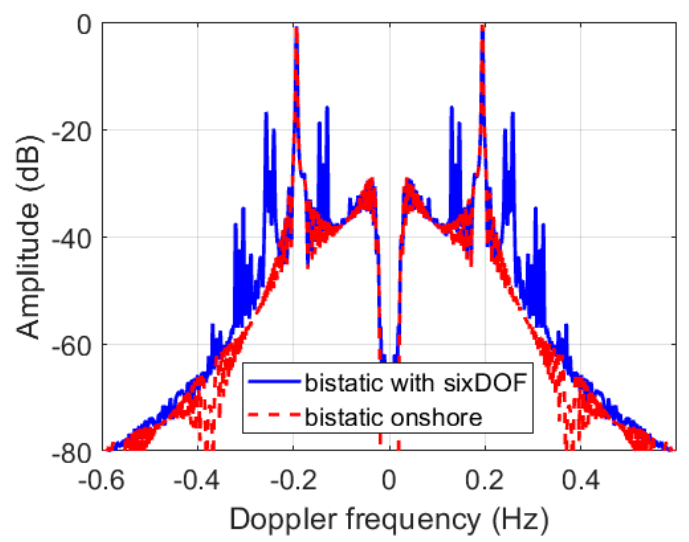

(f)

Figure 7. Simulated first-order RCSs for different motions. (a) Sway case; (b) surge case; (c) yaw case; (d) pitch case; (e) roll case; (f) six DOF case.

Figure 8 shows the simulated second-order RCSs for different platform motion models. The additional motion-induced peaks appearing in the second-order RCS curve are not obvious. Similar to the sway case, the effect of surge on the second-order RCS is also small, as shown in Figure 8b. From Figure $8 \mathrm{~d}$,e, the effect of pitch and roll on the second-order RCSs may be ignored. However, yaw has an important effect on the second-order RCS due to a larger displacement of the antenna, as shown in Figure $8 \mathrm{c}$. When a six DOF oscillation motion model is considered, more additional peaks caused by 
each one-dimensional oscillation motion and the combined motion may appear in the second-order RCS curve and the corresponding frequency locations are

$$
\omega_{d}=\omega_{h d}+\sum_{i=1}^{2} n_{i} \omega_{i}+\sum_{i=3}^{5}\left(2 n_{i 1}+n_{i 2}\right) \omega_{i}
$$

and

$$
\omega_{d}=\omega_{e d}+\sum_{i=1}^{2} n_{i} \omega_{i}+\sum_{i=3}^{5}\left(2 n_{i 1}+n_{i 2}\right) \omega_{i} .
$$

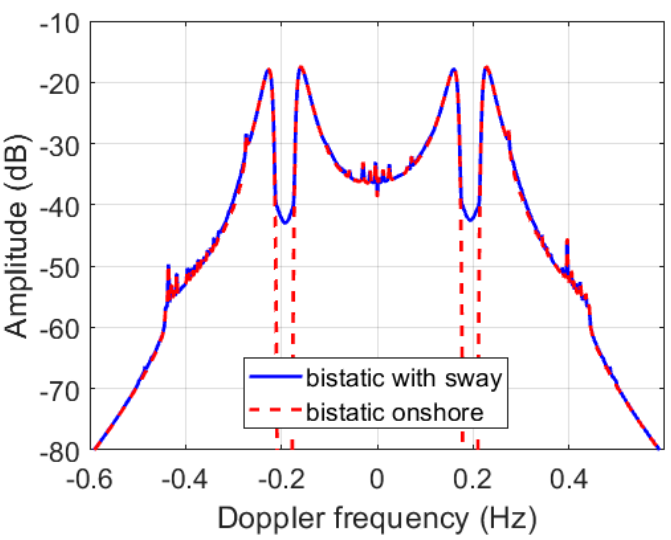

(a)

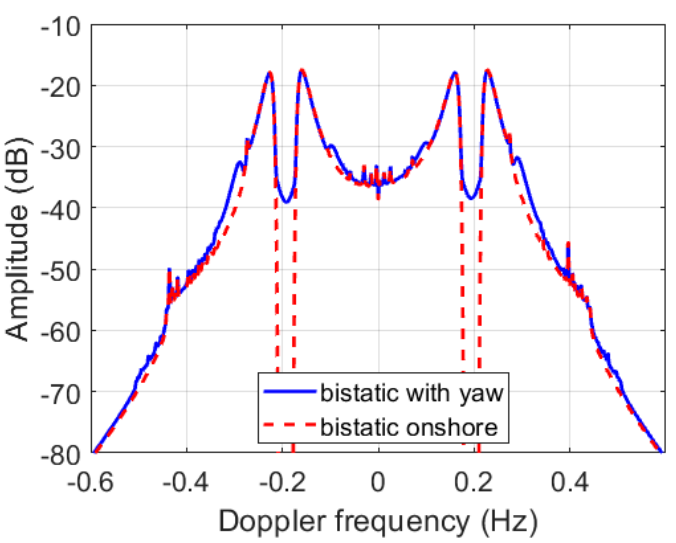

(c)

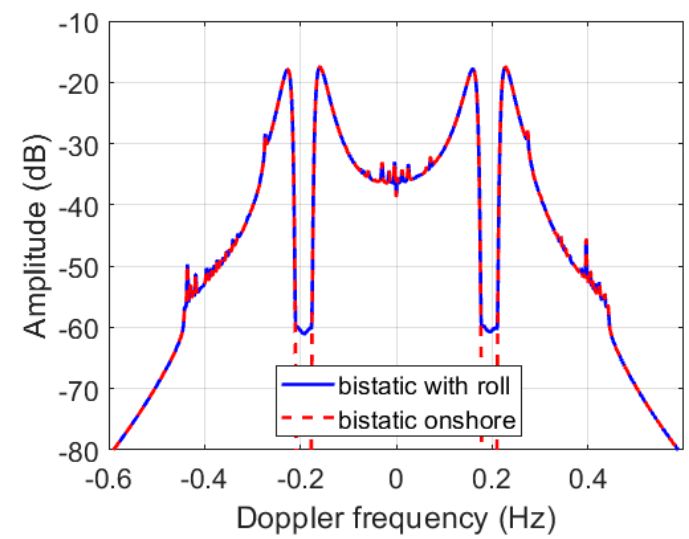

(e)

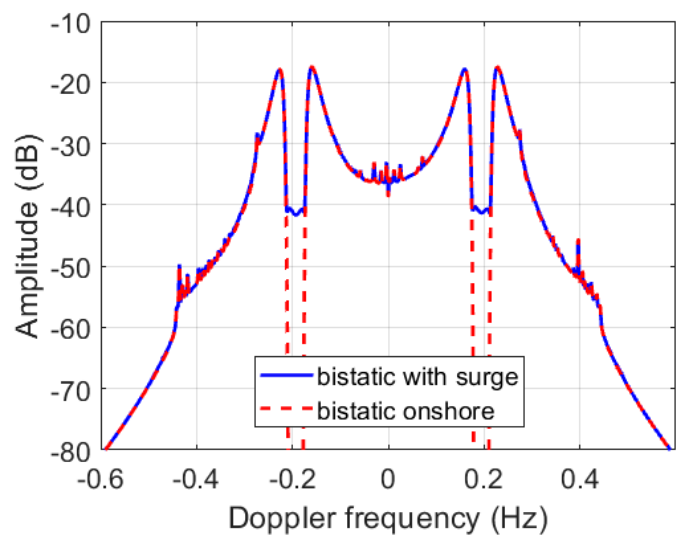

(b)

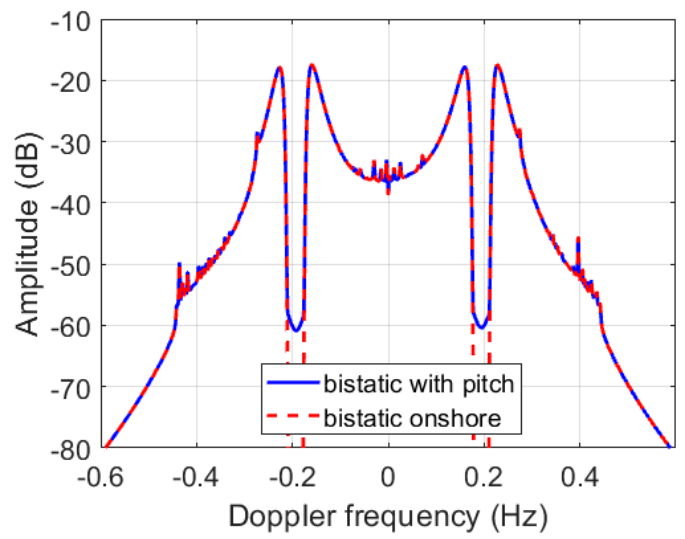

(d)

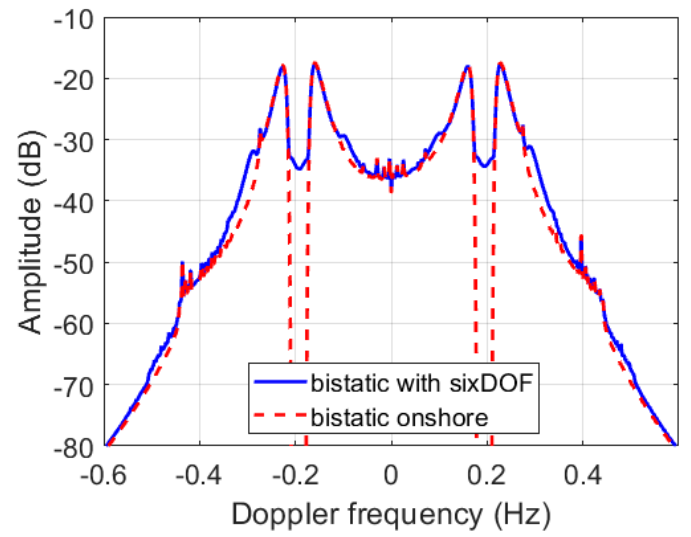

$(\mathbf{f})$

Figure 8. Simulated second-order RCSs for different motions. (a) Sway case; (b) surge case; (c) yaw case; (d) pitch case; (e) roll case; (f) six DOF case. 
However, by comparing Figure $8 \mathrm{c}, \mathrm{f}$, the RCS curves are basically similar. That is, the modulation effect of yaw on the second-order RCS is dominant.

Figure 9 displays the simulated total RCS containing the first- and second-order RCSs for the bistatic HF radar incorporating a single-frequency six DOF oscillation motion model. From Figure 9, it is seen that the motion-induced peaks appearing in the first-order RCS curve will overlap with the second-order RCS curve and, then, the amplitude of the second-order RCS may be raised. For such a case, the amplitudes of the Bragg peaks are still larger than those of the motion-induced peaks. Compared to the sway case in Figure 6b, more motion-induced peaks with larger amplitude appear in the total RCS curve. Therefore, in practice, just considering one- or two-dimensional oscillation motion is not realistic.

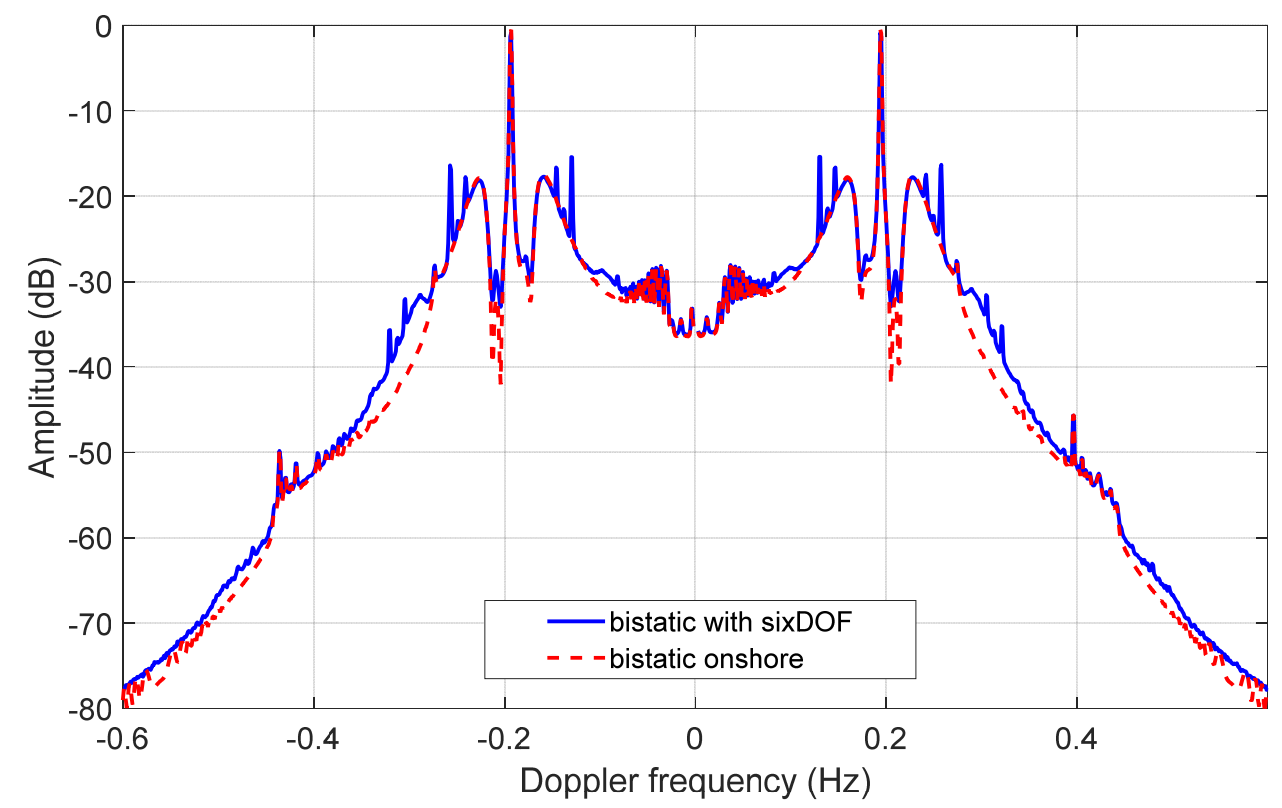

Figure 9. Simulated total RCSs containing first- and second-order RCSs.

\subsection{Effect of Dual-Frequency Model on RCS}

In an actual marine environment, the frequency of the oscillation motion of a floating platform varies from low frequency (LF) to WF [40]. The WF response at the WF is $0.2-2 \mathrm{rad} / \mathrm{s}$ and the LF response at the in-plane resonances is around $0.02 \mathrm{rad} / \mathrm{s}$. Therefore, in order to interpret the characteristics of the sea echo signals more realistically, a multi-frequency six DOF oscillation motion model incorporating LF and WF should be considered. In this study, in order to simply the simulation, a dual-frequency six DOF oscillation motion model incorporating both LF and WF was used. The LF six DOF oscillation motion parameters corresponding to the maximum spectral density of each one-dimensional oscillation motion were selected to simulate RCSs, which are listed in Table 2.

Table 2. Six DOF oscillation motion parameters for low frequency (LF).

\begin{tabular}{llllll}
\hline Parameters & Sway & Surge & Yaw & Pitch & Roll \\
\hline Frequency (rad/s) & 0.04 & 0.05 & 0.03 & 0.03 & 0.03 \\
Amplitude & $4.32 \mathrm{~m}$ & $4.27 \mathrm{~m}$ & $6.87^{\circ}$ & $0.2^{\circ}$ & $0.24^{\circ}$ \\
\hline
\end{tabular}


Similar to the WF case, six DOF oscillation motion with LF will also result in some additional peaks. The frequency locations of these motion-induced peaks are extremely close to the Bragg peaks because of a low oscillation motion frequency. When a dual-frequency six DOF oscillation motion model incorporating a LF model and a WF model is considered, the frequency locations of the motion-induced peaks in the first- and second-order RCS curves are, respectively,

$$
\begin{aligned}
& \omega_{d}=\omega_{b B}+\sum_{i=1}^{2}\left[\sum_{j=1}^{N_{i}} n_{i, j} \omega_{i, j}\right]+\sum_{i=3}^{5}\left[\sum_{j=1}^{N_{i}}\left(2 n_{i 1, j}+n_{i 2, j}\right) \omega_{i, j}\right], \\
& \omega_{d}=\omega_{h d}+\sum_{i=1}^{2}\left[\sum_{j=1}^{N_{i}} n_{i, j} \omega_{i, j}\right]+\sum_{i=3}^{5}\left[\sum_{j=1}^{N_{i}}\left(2 n_{i 1, j}+n_{i 2, j}\right) \omega_{i, j}\right],
\end{aligned}
$$

and

$$
\omega_{d}=\omega_{e d}+\sum_{i=1}^{2}\left[\sum_{j=1}^{N_{i}} n_{i, j} \omega_{i, j}\right]+\sum_{i=3}^{5}\left[\sum_{j=1}^{N_{i}}\left(2 n_{i 1, j}+n_{i 2, j}\right) \omega_{i, j}\right],
$$

where $N_{i}=2$ represents two frequency components. Figure 10 displays the simulated first- and second-order RCSs for bistatic HF radar incorporating a dual-frequency six DOF oscillation motion model. From Figure 10a, the LF motion-induced peaks appear not only near the Bragg peaks but also near the WF motion-induced peaks, which agrees well with Equation (62). Furthermore, the amplitudes of the Bragg peaks and the WF motion-induced peaks are lower than those of the LF motion-induced peaks due to the modulation effect, which may 'break' Bragg scatter mechanism. A comparison of Figures $8 \mathrm{f}$ and $10 \mathrm{~b}$ shows that the WF motion is the dominant factor affecting the second-order RCS. Figure 10c shows the total RCS containing the first- and second-order RCSs. From Figure 10c, it can be seen that a dual-frequency six DOF oscillation motion may have a more significant effect than a single-frequency case in Figure 9. In addition, the effects of different wind directions, wind speeds, and radar parameters on RCS for bistatic HF radar incorporating a dual-frequency six DOF oscillation motion model are similar to the sway case $[30,31]$ and are not further discussed here.

\subsection{Effect of Bistatic Angle on RCS}

Figure 11 shows the simulated total RCSs containing the first- and second-order RCSs for different bistatic angles. It is obvious that the Bragg peaks, both the hydrodynamic and electromagnetic peaks for the second-order scatter and additional peaks caused by six DOF oscillation motion move closer to zero Doppler frequency while the bistatic angle is increasing. Furthermore, as the bistatic angle increases, the amplitudes of the second-order RCSs decrease, and the modulation effect caused by the platform motion is weakened. It should be noted that, from Equation (58), the second-order electromagnetic peaks may be far away from the Bragg peaks or even diminished from the total RCS curve for a large bistatic angle, for example $\phi_{0}=85^{\circ}$ as shown in Figure 11. 


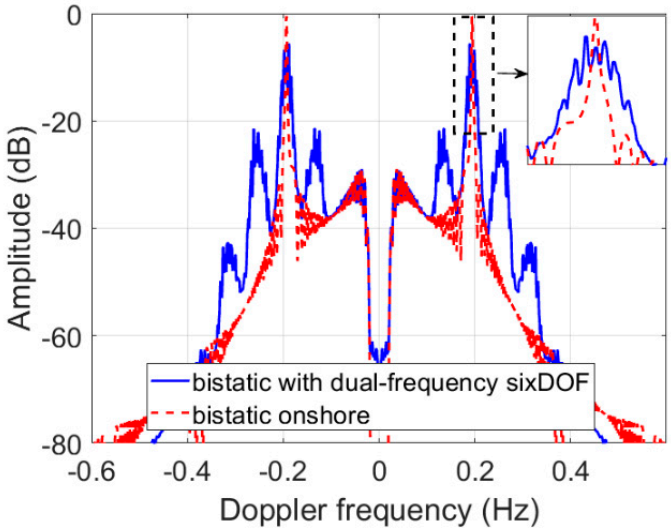

(a)

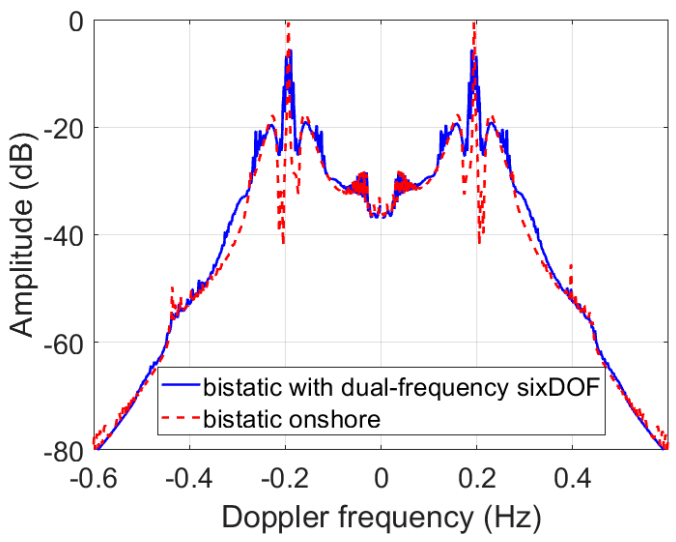

(c)

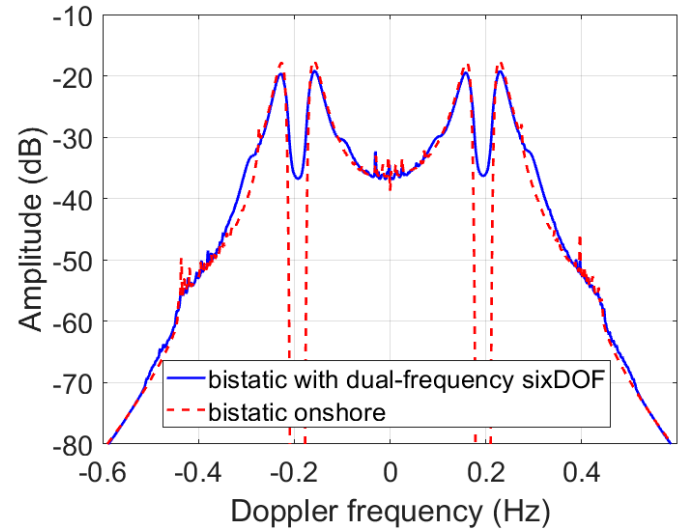

(b)

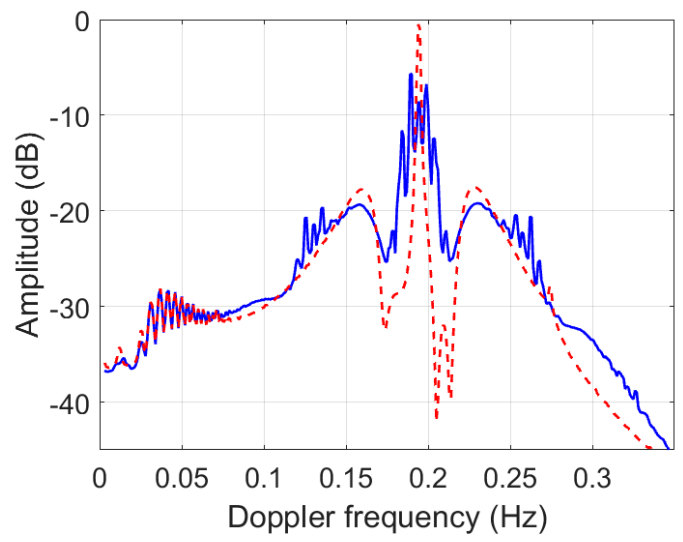

(d)

Figure 10. Simulated RCSs for a dual-frequency six DOF oscillation motion model. (a) First-order RCS; (b) second-order RCS; (c) total RCS; (d) zoomed in view of the positive Doppler frequency in (c).

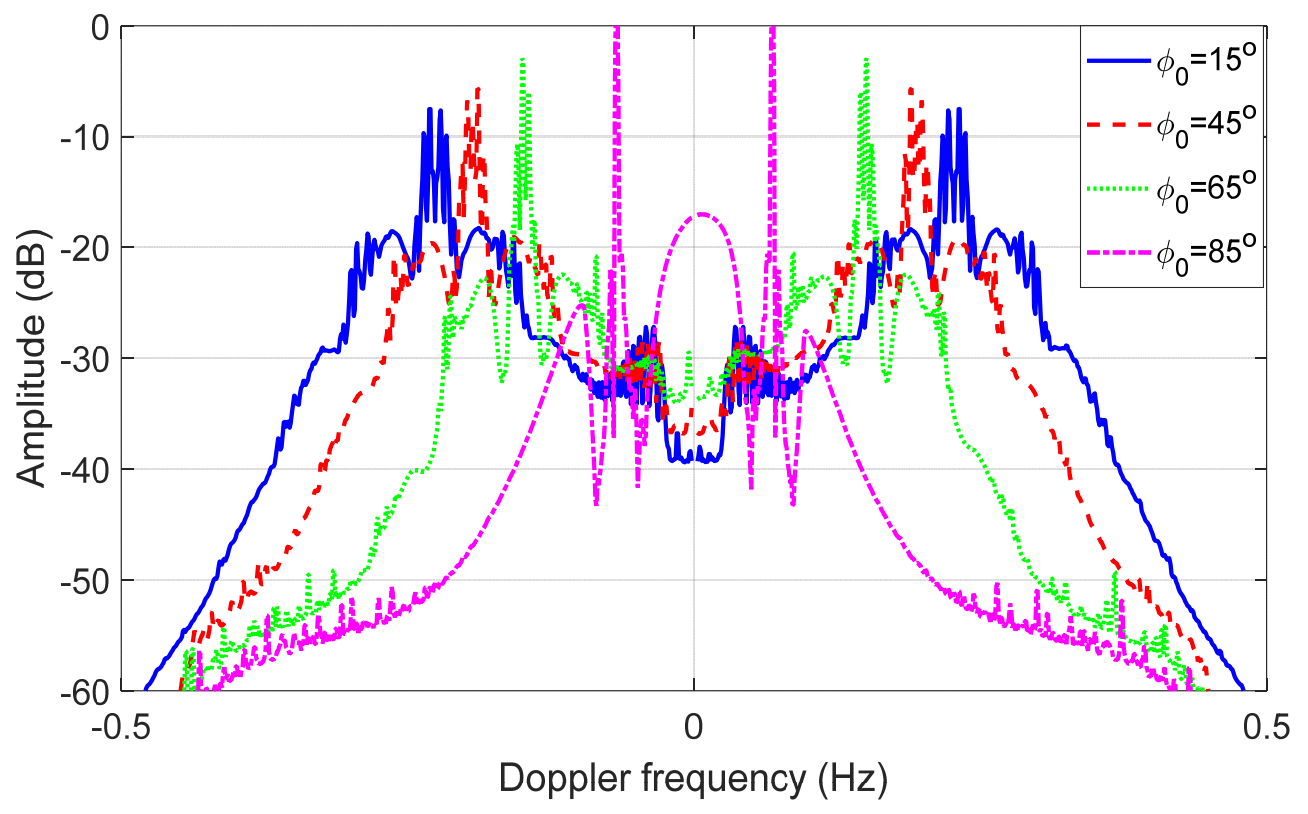

Figure 11. Simulated total RCSs containing first- and second-order RCSs for different bistatic angles.

\section{Discussion}

On the basis of the preceding simulation results, it may be clearly concluded that a dual-frequency six DOF oscillation motion has a critical effect on ocean surface scattering cross section of bistatic HF 
radar, which may seriously affect the applications of the bistatic HF radar installed on a floating platform in ocean surface moving target detection and remote sensing of ocean surface dynamics parameters such as wind direction, wind speed, current, and ocean wave parameters. The characteristics (the energy distributions of the Bragg peaks and motion-induced peaks) of radar Doppler spectra depend on the oscillation motion parameters. Wind speed is also one of the factors affecting the oscillation motion parameters. Although the oscillation motion parameters of a large floating platform under a hurricane condition were selected for examples in this study, a small floating platform may yield similar phenomena under a moderate or low sea state. The reliability still needs to be further verified using field data with different floating platforms (shape and size) and different wind speeds in future work.

The motion-induced peaks may mask the moving target echoes and it may be extremely difficult to separate the target echoes from the motion-induced peaks if the target echoes appear near the Bragg peaks. This is because the amplitudes of the motion-induced peaks may be larger than those of the Bragg peaks, which may cause false alarm.

Furthermore, ocean surface wind direction is generally extracted according to the intensity ratio of the positive and negative Bragg peaks. Considering that the energies of the Bragg peaks are modulated by six DOF oscillation motion, the wind direction measurement results may be inaccurate if the modulation effect is ignored. Ocean surface current is generally measured based on the position difference between the theoretical Bragg peaks without ocean surface current and the measured Bragg peaks with ocean surface current. Generally, for the field data, the positions of the Bragg peaks are identified by searching for the strongest peaks in the positive and negative Doppler spectrum. However, due to the modulation effect of six DOF oscillation motion, the energies of the Bragg peaks may be lower than those of the motion-induced peaks, which may severely influence the measurement of ocean surface current. As we all know, the information of ocean surface wind speed and wave parameters is contained in the second-order radar Doppler spectrum. Although the modulation effect of six DOF oscillation motion on the second-order RCS is small, the motion-induced peaks in the first-order RCS may overlap with the second-order RCS, which would severely contaminate the second-order RCS and may have an unfavorable effect on the measurement of wind speed and ocean wave parameters.

Moreover, for six DOF oscillation motion, yaw is a dominant factor affecting RCS. Considering that, the oscillation motion can be regarded as frequency modulation on RCS and the modulation effect depends on the amplitude and frequency of the oscillation motion. Therefore, in order to reduce the effect of yaw on RCS and considering the influence of the floating platform superstructure on electromagnetic scattering, the installation location of the antenna should be at the edge of the floating platform but near the rotation center.

In addition, the modulation effect of six DOF oscillation motion on RCS is reduced with increased bistatic angle. That is, the amplitudes of the motion-induced peaks and the second-order RCS decrease for a large bistatic angle, which may be beneficial for ocean surface moving target detection. However, this case may be adverse for the extraction of ocean surface wind speed and wave parameters from the second-order RCS due to the disappearance of the second-order electromagnetic peaks for a large bistatic angle. Therefore, it is very important to choose a reasonable bistatic angle for different application purposes using a floating platform based bistatic HF radar.

Therefore, in order to improve the performance of moving target detection and the accuracies of ocean surface dynamics parameter measurements, a motion compensation method should be investigated to remove the motion-induced peaks in RCS and to recover the amplitudes of the firstand second-order RCSs in the future.

\section{Conclusions}

In this paper, the first- and second-order ocean surface cross sections for bistatic HF radar incorporating a multi-frequency six DOF oscillation motion model were theoretically derived. When the bistatic angle is zero, the derived results can be reduced to the monostatic case, and when there 
is no six DOF oscillation motion, the derived results can be simplified to the onshore bistatic case. Simulations were conducted under different oscillation motion models and different bistatic angles.

Results show that each one-dimensional oscillation motion may induce additional peaks symmetrically appearing the first- and second-order radar Doppler spectra and the combined six DOF oscillation motion may result in more additional peaks. The amplitudes and frequencies of these motion-induced peaks depend on the amplitude and frequency of six DOF oscillation motion. The platform oscillation motion can be viewed as frequency modulation for radar echoes and the modulation effect of six DOF oscillation motion on the first-order radar Doppler spectra is more obvious than that on the second-order radar Doppler spectra. However, the motion-induced peaks appearing in the first-order radar spectra may overlap with the second-order radar spectra, which may raise the second-order radar spectra. It should be noted that yaw is the dominant factor affecting radar Doppler spectra, especially for the second-order spectra. Furthermore, the amplitudes of the Bragg peaks may be lower than those of the motion-induced peaks if a LF six DOF oscillation motion model is considered. This is a very important phenomenon for the application of bistatic HF radar. In addition, the modulation effect of six DOF oscillation motion and amplitudes of the second-order radar Doppler spectra decrease with increasing bistatic angle. For a large bistatic angle, the second-order electromagnetic peaks may be far away from the Bragg peaks or even diminished from radar Doppler spectra. Therefore, if the influences of the platform oscillation motion and bistatic angle on radar Doppler spectra are ignored, it will severely affect the applications of bistatic HF radar in moving target detection and ocean surface dynamics parameter measurements.

Here, the derived results were investigated only with simulated data, the rationality of the derived results should be further validated with field data. Nonetheless, this work provides an important theoretical foundation to determine suitable geometries for the deployment of a platform-based bistatic HF radar.

Author Contributions: Conceptualization, G.Y., J.X.; Methodology, G.Y., J.X., W.H.; Writing-original draft preparation, G.Y.; Writing—review and editing, J.X., W.H.; Software, G.Y.

Funding: This research was funded by National Natural Science Foundation of China key project (61132005), Special Research Foundation for Harbin Science and Technology Innovation Talents (RC2014XK009022), Natural Sciences and Engineering Research Council of Canada Discovery Grants under Grant NSERC RGPIN-2017-04508 and Grant RGPAS-2017-507962.

Acknowledgments: The authors would like to thank anonymous reviewers for their valuable comments in improving the quality of this paper.

Conflicts of Interest: The authors declare no conflict of interest.

\section{References}

1. Barrick, D.E. Extraction of wave parameters from measured HF radar sea-echo Doppler spectra. Radio Sci. 1986, 12, 415-424. [CrossRef]

2. Lipa, B.J.; Barrick, D.E. Extraction of sea state from HF radar sea echo: Mathematical theory and modeling. Radio Sci. 1986, 21, 81-100. [CrossRef]

3. Khan, R.; Gamberg, B.; Power, D.; Walsh, J.; Dawe, B.; Pearson, W.; Millan, D. Target detection and tracking with a high frequency ground wave radar. IEEE J. Ocean. Eng. 1994, 19, 540-548. [CrossRef]

4. Hisaki, Y. Nonlinear inversion of the integral equation to estimate ocean wave spectra from HF radar. Radio Sci. 1996, 31, 25-39. [CrossRef]

5. Heron, M.L.; Prytz, A. Wave height and widn direction from the HF coastal ocean surface radar. Can. J. Remote Sens. 2002, 28, 385-393. [CrossRef]

6. Huang, W.; Wu, S.; Gill, E.W.; Wen, B.; Hou, J. HF radar wind and wave measurement over the Eastern China Sea. IEEE Trans. Geosci. Reomote Sens. 2002, 40, 1950-1955. [CrossRef]

7. Emery, B.M.; Washburn, L.; Harlan, J.A. Evaluating radial current measurements from CODAR high-frequency radars with moored current meters. J. Atmos. Ocean. Technol. 2004, 21, 1259-1271. [CrossRef]

8. Huang, W.; Gill, E.W.; Wu, S.; Wen, B.; Yang, Z.; Hou, J. Measuring surface wind direction by monostatic HF ground-wave radar at the Eastern China Sea. IEEE J. Ocean. Eng. 2004, 29, 1032-1037. [CrossRef] 
9. Wyatt, L.R.; Green, J.J.; Middleditch, A.; Moorhead, M.D.; Howarth, J.; Holt, M.; Keogh, S. Operational wave, current, and wind measurements with the pisces HF radar. IEEE J. Ocean. Eng. 2006, 31, 819-834. [CrossRef]

10. Jangal, F.; Saillant, S.; Helier, M. Wavelet contribution to remote sensing of the sea and target detection for a high-frequency surface wave radar. IEEE Geosci. Remote Sens. Lett. 2008, 5, 552-556. [CrossRef]

11. Li, Q.; Zhang, W.; Li, M.; Niu, J.; Wu, Q.M.J. Automatic detection of ship targets based on wavelet transform for HF surface wavelet radar. IEEE Geosci. Remote Sens. Lett. 2017, 14, 714-718. [CrossRef]

12. Tian, Y.; Wen, B.; Zhou, H.; Wang, C.; Yang, J.; Huang, W. Wave Height Estimation from First-Order Backscatter of a Dual-Frequency High Frequency Radar. Remote Sens. 2017, 9, 1186. [CrossRef]

13. Shen, W.; Gurgel, K.W. Wind Direction Inversion from Narrow-Beam HF Radar Backscatter Signals in Low and High Wind Conditions at Different Radar Frequencies. Remote Sens. 2018, 10, 1480. [CrossRef]

14. Walsh, J.; Gill, E.W. An analysis of the scattering of high-frequency electromagnetic radiation form rough surfaces with application to pulse radar operating in backscatter mode. Radio Sci. 2000, 35, 1337-1359. [CrossRef]

15. Gill, E.W.; Walsh, J. High-frequency bistatic cross sections of the ocean surface. Radio Sci. 2001, 36, 1459-1475. [CrossRef]

16. Gill, E.W.; Huang, W.; Walsh, J. The effect of bistatic scattering angle on the high frequency radar cross sections of the ocean surface. IEEE Geosci. Remote Sens. Lett. 2008, 5, 143-146. [CrossRef]

17. Lipa, B.J.; Whelan, C.; Rector, B.; Moorhead, M.D.; Howarth, J.; Holt, M.; Keogh, S. HF radar bistatic measurement of surface current velocities: Drifter comparisons and radar consistency checks. Remote Sens. 2009, 1, 1190-1211. [CrossRef]

18. Trizna, D.B. A bistatic HF radar for current mapping and robust ship tracking. Sea Technol. 2009, 50, $29-33$.

19. Grosdidier, S.; Forget, P.; Barbin, Y.; Cuerin, C.A. HF bistatic ocean Doppler spectra: Simulation versus experimentation. IEEE Trans. Geosci. Reomote Sens. 2014, 52, 2138-2148. [CrossRef]

20. Huang, W.; Gill, E.W.; Wu, X.; Li, L. Measurement of sea surface wind direction using bistatic high-frequency radar. IEEE Trans. Geosci. Reomote Sens. 2012, 50, 4117-4122. [CrossRef]

21. Zhang, J.; Gill, E.W. Extraction of ocean wave spectra from simulated noisy bistatic high-frequency radar data. IEEE J. Ocean. Eng. 2006, 31, 779-796. [CrossRef]

22. Silva, M.T.; Shahidi, R.; Gill, E.W.; Huang, W. Nonlinear extraction of directional ocean wave spectrum from synthetic bistatic high-frequency surface wave radar data. IEEE J. Ocean. Eng. 2019, in press. [CrossRef]

23. Lipa, B.J.; Barrick, D.E.; Isaacson, J.; Lilleboe, P.M. CODAR wave measurements from a North Sea semisubmersible. IEEE J. Ocean. Eng. 1990, 15, 119-225. [CrossRef]

24. Howell, R.; Walsh, J. Measurement of ocean wave spectra using a ship-mounted HF radar. IEEE J. Ocean. Eng. 1993, 18, 306-310. [CrossRef]

25. Gurgel, K.W.; Essen, H. On the performance of a shipborne current mapping HF radar. IEEE J. Ocean. Eng. 2000, 25, 183-191. [CrossRef]

26. Xie, J.; Yuan, Y.; Liu, Y. Experimental analysis of sea clutter in shipborne HFSWR. IEE Proc.-Radar Sonar Navig. 2001, 148, 67-71. [CrossRef]

27. Walsh, J.; Huang, W.; Gill, E.G. The first-order high frequency radar ocean surface cross section for an antenna on a floating platform. IEEE Trans. Antennas Propag. 2010, 58, 2994-3003. [CrossRef]

28. Walsh, J.; Huang, W.; Gill, E.G. The second-order high frequency radar ocean surface cross section for an antenna on a floating platform. IEEE Trans. Antennas Propag. 2012, 60, 4804-4813. [CrossRef]

29. Sun, M.; Xie, J.; Ji, Z.; Yao, G. Ocean surface cross sections for shipborne HFSWR with sway motion. Radio Sci. 2016, 51, 1745-1757. [CrossRef]

30. Ma, Y.; Gill, E.W.; Huang, W. First-order bistatic high frequency radar ocean surface cross section for an antenna on a floating platform. IET Radar Sonar Navig. 2016, 10, 1136-1144. [CrossRef]

31. Ma, Y.; Huang, W.; Gill, E.W. The second-order bistatic high frequency radar ocean surface cross section for an antenna on a floating platform. Can. J. Remote Sens. 2016, 42, 332-343. [CrossRef]

32. Das, S.N.; Das, S.K. Mathematical modeling of sway, roll and yaw motions: Order-wise analysis to determine coupled characteristics and numerical simulation for restoring moment's sensitivity analysis. Acta Mech. 2010, 213, 305-322. [CrossRef]

33. Tahar, A.; Kim, M.H. Hull/mooring/riser coupled dynamics analysis and sensitivity study of tanker-based FPSO. Appl. Ocean Res. 2003, 25, 367-382. [CrossRef] 
34. Ma, Y.; Gill, E.W.; Huang, W. Bistatic high-frequency radar ocean surface cross section incorporating a dual-frequency platform motion model. IEEE J. Ocean. Eng. 2018, 43, 205-210. [CrossRef]

35. Yao, G.; Xie, J.; Huang, W. First-order ocean surface cross-section for shipborne HFSWR incorporating a horizontal oscillation motion model. IET Radar Sonar Navig. 2018, 41, 970-981. [CrossRef]

36. Yao, G.; Xie, J.; Huang, W. First-order ocean surface cross section for bistatic HFSWR incorporating a horizontal oscillation motion model. In Proceedings of the IEEE Radar Conference, Boston, MA, USA, 22-26 April 2019.

37. Hasselmann, K. On the nonlinear energy transfer in a gravity wave spectrum, part 1, general theory. J. Fluid Mech. 1962, 12, 481-500. [CrossRef]

38. Pierson, W.J.; Moskowitz, L. A proposed spectral form for fully developed wind sea based on the similarity theory of S. A. Kitaigorodskii. J. Geophys. Res. 1964, 69, 5181-5190. [CrossRef]

39. Longuet-Higgins, M.S.; Cartwright, D.E.; Smith, N.D. Observation of the directional spectrum of sea waves using the motion of a floating buoy. In Proceedings of the Conference Ocean Wave Spectra; Prentice-Hall Inc.: Englewood Cliffs, NJ, USA, 1963; pp. 111-136.

40. Low, Y.M.; Langley, R.S. Time and frequency domain coupled analysis of deepwater floating production systems. Appl. Ocean Res. 2006, 28, 371-385. [CrossRef]

(C) 2019 by the authors. Licensee MDPI, Basel, Switzerland. This article is an open access article distributed under the terms and conditions of the Creative Commons Attribution (CC BY) license (http://creativecommons.org/licenses/by/4.0/). 